\title{
Oscillating multiple giants
}

\author{
Ryo Suzuki \\ Shing-Tung Yau Center of Southeast University, \\ 15th Floor, Yifu Architecture Building, No.2 Sipailou, \\ Xuanwu district, Nanjing, Jiangsu, 210096, China \\ E-mail: rsuzuki.mp@gmail.com
}

Abstract: We propose a new example of the AdS/CFT correspondence between the system of multiple giant gravitons in $A d S_{5} \times S^{5}$ and the operators with $O\left(N_{c}\right)$ dimensions in $\mathcal{N}=4$ super Yang-Mills. We first extend the mixing of huge operators on the Gauss graph basis in the $\mathfrak{s u}(2)$ sector to all loops of the 't Hooft coupling, by demanding the commutation of perturbative Hamiltonians in an effective $\mathrm{U}(p)$ theory, where $p$ corresponds to the number of giant gravitons. The all-loop dispersion relation remains gapless at any $\lambda$, which suggests that harmonic oscillators of the effective $\mathrm{U}(p)$ theory should correspond to the classical motion of the D3-brane that is continuously connected to non-maximal giant gravitons.

KEYworDs: 1/N Expansion, AdS-CFT Correspondence, D-branes

ArXiv EPrint: 2101.05310 


\section{Contents}

1 Introduction 1

2 Mixing of huge operators in $\mathcal{N}=4$ SYM 3

2.1 Perturbative dilatation operator 3

2.2 Commutation relations 5

2.3 Continuum limit 6

3 All-loop ansatz $\quad 7$

3.1 Constraints on higher-loop dilatations 8

$\begin{array}{ll}3.2 \text { Continuum limit at higher loops } & 10\end{array}$

4 Strong coupling $\quad 11$

$\begin{array}{lll}4.1 & \text { D3-brane action } & 12\end{array}$

$\begin{array}{lll}4.2 & \text { Classical solutions } & 13\end{array}$

4.2.1 Ground state 13

$\begin{array}{lll}4.2 .2 & \text { Excited states } & 13\end{array}$

$\begin{array}{lll}\text { 4.2.3 Excited states at higher orders } & 15\end{array}$

5 Comments on AdS/CFT $\quad 17$

5.1 Comparison with the oscillating D3-brane 17

$\begin{array}{ll}5.2 \text { On reflecting magnons } & 19\end{array}$

$\begin{array}{lll}6 & \text { Summary } & 21\end{array}$

A Review of the Gauss graph basis $\quad 22$

$\begin{array}{lll}\text { A.1 Notation } & 22\end{array}$

$\begin{array}{lll}\text { A.2 } & \text { Distant corners approximation } & 23\end{array}$

A.3 Gauss graph basis 24

$\begin{array}{lll}\text { A.3.1 Skew Young diagrams } & 24\end{array}$

$\begin{array}{lll}\text { A.3.2 } & \text { Adjacency matrix } & 25\end{array}$

A.3.3 Permutation and the double coset 25

A.3.4 Operator mixing in the Gauss graph basis 26

B Explicit one-loop spectrum $\quad 27$

$\begin{array}{ll}\text { B.1 Continuum case } & 27\end{array}$

$\begin{array}{lll}\text { B.2 Discrete case } & 29\end{array}$

B.3 Examples of the eigenvalues 32

C Identities of hypergeometric functions 33 
D Details of strong coupling analysis

D.1 Geometry 34

$\begin{array}{lll}\text { D.2 } & \text { Spherical harmonics } & 34\end{array}$

D.3 Classical solutions at $j=0,1 \quad 35$

$\begin{array}{lll}\text { E On non-abelian DBI } & 36\end{array}$

\section{Introduction}

Traditionally the AdS/CFT correspondence has been studied in the planar large $N_{c}$ limit [1]. Whether AdS/CFT holds true in a non-planar but still large $N_{c}$ limit is a challenging question. Such a nontrivial limit can be implemented by deforming the background theory or spacetime, or by introducing semiclassical objects carrying the dimensions or energy of order $N_{c}$.

One of the most studied examples of AdS/CFT is the correspondence between string theory on $\mathrm{AdS}_{5} \times \mathrm{S}^{5}$ and $\mathcal{N}=4$ super Yang-Mills (SYM) theory. We depart from the planar region of $\mathcal{N}=4 \mathrm{SYM}$ by studying huge operators whose dimensions are comparable to the rank of the gauge group $N_{c} \cdot{ }^{1}$ The operators with $O\left(N_{c}^{2}\right)$ dimensions correspond to the Lin-Lunin-Maldacena (LLM) geometry at strong coupling [3], while those with $O\left(N_{c}\right)$ dimensions correspond to the giant graviton [4]. This correspondence continues to non-BPS operators in both of the $O\left(N_{c}^{2}\right)$ and $O\left(N_{c}\right)$ cases. For the former case, an isomorphism between non-BPS states was conjectured between the LLM geometry and $\mathcal{N}=4$ SYM [5-9]. For the latter case, non-BPS states around the giant graviton are less well-understood. This is the main subject of this paper.

Let us first review recent progress on the weak coupling side.

In AdS/CFT, the half-BPS operators with huge dimensions should be organized through the operator basis labeled by a Young diagram [10]. Similarly, a convenient way to describe non-BPS operators with huge dimensions is the restricted Schur basis, labeled by a set of Young diagrams $[11,12]$. The dilatation operator expressed in this basis mixes the Young diagrams with different shapes.

For simplicity, consider the operators in the $\mathfrak{s u}(2)$ sector, which consists of complex scalars $Z$ and $Y$ of $\mathcal{N}=4 \mathrm{SYM}$. Suppose that a small number of $Y$ 's are added to a large number of $Z$ 's. If the Young diagram representing $Z$ 's has $p$ long columns, this type of operators roughly corresponds to a system of $p$ spherical giant gravitons in $\operatorname{AdS}_{5} \times \mathrm{S}^{5}$. The Young diagram representing $Y$ 's describes a small fluctuation of the giant gravitons. ${ }^{2}$

The one-loop mixing in this setup is remarkably simple. First, the number of columns $p$ does not change at large $N_{c}$, because giant gravitons are semi-classical objects at strong

\footnotetext{
${ }^{1}$ Another promising approach to a non-planar large $N_{c}$ limit is the localization, which is valid at any $N_{c}$ and can extract non-BPS data [2].

${ }^{2}$ Here $Y$ 's and $Z$ 's constitute a huge Young diagram whose shape wildly fluctuates due to the operator mixing. This situation is different from a single-trace operator coupled to $\operatorname{det}(Z)$, where the operator mixing does not spoil the color structure at the leading order of large $N_{c}$.
} 
coupling [13]. Second, the operator mixing splits into the mixing of $Z$ 's and the mixing of $Y$ 's. Third, the one-loop spectrum eventually reduces to a set of $p$ decoupled harmonic oscillators $[14,15]$. The last observation is called the non-planar integrability in the literature.

We should emphasize that the mixing problem of huge operators is quite different from the planar mixing problem, and the development of sophisticated techniques has been crucial. The mixing problem of $Y$ 's is solved by the Gauss graph basis, which counts the number of open strings ending on different giant graviton branes [16]. The same technique can be used to simplify the mixing in the $\mathfrak{s u}(3)$ and $\mathfrak{s u}(2 \mid 3)$ sectors $[17,18]$. Explicit computation of the mixing matrix has been given up to two loops in $g_{\mathrm{YM}}^{2}$ in [19]. There are also trial studies at higher loops [20,21]. More generally, the mixing problem corresponding to $p$ giant gravitons can be described by an effective $\mathrm{U}(p)$ theory $[22,23]$. The Hamiltonian of the effective $\mathrm{U}(p)$ theory has the symmetry $\mathrm{U}(1)^{p}$ in the distant corners approximation, namely when the differences of the length of the adjacent columns are large.

Next, the key developments on the strong coupling side are summarized.

The D-brane motion is described by a low energy effective action which consists of Dirac-Born-Infeld (DBI) and Chern-Simons (CS) terms [24]. The giant graviton is a classical solution of the D3-brane action moving in the $\mathrm{AdS}_{5} \times \mathrm{S}^{5}$ background. The spherical giant graviton wraps $\mathrm{S}^{3}$ inside $\mathrm{S}^{5}$ [4], and the AdS giant wraps $\mathrm{S}^{3}$ inside $\mathrm{AdS}_{5}[25,26]$. The quantum fluctuation modes around the giant graviton have been studied in [27].

The open strings ending on the giant graviton have been studied from two viewpoints. In the first viewpoint, we replace open strings with $\mathrm{U}(1)$ flux and study the D-brane. The classical motion of a D3-brane in such a background spacetime has been studied in the flat space [28], in the pp-wave [29] and in $\mathrm{AdS}_{5} \times \mathrm{S}^{5}$ [30]. The U(1) gauge fields typically become spiky, and they diverge at the location where open strings end on the D-brane. In the second viewpoint, we study the open string as a classical integrable system [31], or a boundary integrable system [32]. ${ }^{3}$ In both points of view, the brane-string system typically has divergent energy, which is canceled by a divergent angular momentum of open strings, just like the giant magnon [36].

It is expected that the system of open strings with $p$ giant gravitons corresponds to the effective $\mathrm{U}(p)$ theory, but there is still obscurity in this understanding as AdS/CFT. The purpose of this paper is to understand this theory more precisely by revisiting the analysis both in gauge and string theories.

In section 2 and 3, we study the perturbative Hamiltonians of the effective $\mathrm{U}(p)$ theory on the gauge theory side in detail. Possible forms of the effective Hamiltonian are constrained by the GL $(p)$ algebra, and by demanding that the perturbative Hamiltonians at each loop order commute with each other. We find that there are at most $(\ell+1)$ linearly-independent mutually-commuting operators at $\ell$-loops. In the continuum limit, these candidate operators reduce to the harmonic oscillators at one loop, which allows us

\footnotetext{
${ }^{3} \mathrm{~A}$ coherent state description of open strings ending on a mixture of (generally non-maximal) giant gravitons is studied in [33-35].
} 
to conjecture an all-loop ansatz,

$$
\Delta-J=\frac{\tilde{f}(\lambda)}{N_{c}} m n_{12}(\sigma), \quad n_{12}(\sigma) \in \mathbb{Z}_{\geq 0}, \quad m=1,2, \ldots,\left\lceil N_{c}-\frac{n_{Z}}{2}+1\right\rceil
$$

where we put $p=2$ for simplicity, and $\tilde{f}(\lambda)$ is an unknown function of the 't Hooft coupling $\lambda=N_{c} g_{\mathrm{YM}}^{2}$.

Our ansatz (1.1) predicts two remarkable consequences. First, the anomalous dimensions remain non-zero at the leading order of large $N_{c}$, because $m$ can be an integer of $O\left(N_{c}\right)$. Second, the excitations are gapless. Recall that the energy of an open string attached on the $Z=0$ giant gravitons is gapped, because the open string stretching on $\mathrm{S}^{5}$ carries non-zero energy, equal to the length times tension. ${ }^{4}$ This disagreement indicates that the previous analyses on the LLM geometry do not immediately apply to the system of multiple giant gravitons. What does the all-loop ansatz represent at strong coupling?

In section 4 we revisit a classical single D3-brane wrapping $\mathrm{S}^{3}$ inside $\mathrm{AdS}_{5} \times \mathrm{S}^{5}$, and solve the equations of motion around the BPS spherical giant graviton. Following the steps similar to the stability analysis of [27], we found two types of classical solutions oscillating around the BPS giant gravitons. The first type is a point-like D-brane, and the second type is a fuzzy D-brane with non-trivial $\mathrm{KK}$ modes on $\mathrm{S}^{3}$. The energy of the latter solution is

$$
E-J=\frac{N_{c}}{g_{s}}\left[\epsilon^{2} \frac{c_{k}^{2}(k+1)^{2}}{8(1-j)(k+2)}+O\left(\epsilon^{4}\right)\right]
$$

where $c_{k}$ is a numerical constant that remains finite as $k \rightarrow \infty$. We argue that the latter solution (1.2) is a good candidate for the string theory state corresponding to the all-loop harmonic oscillator (1.1) at strong coupling. Our reasoning will be presented in section 5 .

This paper is supplemented by Mathematica files used for the computations in sections 3,4 .

\section{Mixing of huge operators in $\mathcal{N}=4 \mathrm{SYM}$}

We collect known facts about the perturbative mixing of huge operators in $\mathcal{N}=4$ SYM. Our notation and basis facts about the Gauss graph basis are summarized in appendix A.

\subsection{Perturbative dilatation operator}

We express the perturbative dilatation operator in the $\mathfrak{s u}(2)$ sector of $\mathcal{N}=4$ SYM by

$$
D\left(g_{\mathrm{YM}}\right)=\sum_{\ell=0}^{\infty}\left(\frac{g_{\mathrm{YM}}}{4 \pi}\right)^{2 \ell} D_{\ell}
$$

where [37]

$$
\begin{aligned}
& D_{0}=\operatorname{Tr} Y \check{Y}+\operatorname{Tr} Z \check{Z} \\
& D_{1}=-2: \operatorname{Tr}[Y, Z][\check{Y}, \check{Z}]: \\
& D_{2}=-2: \operatorname{Tr}[[Y, Z], \check{Z}][[\check{Y}, \check{Z}], Z]:-2: \operatorname{Tr}[[Y, Z], \check{Y}][[\check{Y}, \check{Z}], Y]:-2\left(N_{c}-1\right) D_{1} .
\end{aligned}
$$

\footnotetext{
${ }^{4}$ Here the energy is measured in the unit of string tension $\sqrt{\lambda}$ and not in the D3-brane tension $N_{c} / g_{s} \sim$ $N_{c}^{2} / \lambda$. The string with a finite length produces a gap in the dispersion relation, even if $g_{s} \ll 1$ or $\lambda \ll N_{c}$.
} 
The fields $(\Phi, \check{\Phi})$ satisfy the $U\left(N_{c}\right)$ Wick rules

$$
\operatorname{Tr}(A \check{\Phi} B \Phi)=\operatorname{Tr}(A) \operatorname{Tr}(B), \quad \operatorname{Tr}(A \check{\Phi}) \operatorname{Tr}(B \Phi)=\operatorname{Tr}(A B), \quad \operatorname{Tr}(1)=N_{c} .
$$

The explicit form of $D_{\ell}$ has been known up to five loops [38, 39],

We assume $n_{Z}=O\left(N_{c}\right), n_{Y}=O(1)$. The operators in the Gauss graph basis are denoted by

$$
O^{R, r}(\sigma)=O(\vec{l})=O\left(l_{1}, l_{2}, \ldots, l_{p}\right)
$$

as in (A.20), where $\vec{l}$ specifies the Young diagram for $Z$. Let us write $D_{\ell}$ acting on the Gauss graph operators by $D_{\ell}^{G}$. At the leading order of large $N_{c}$, these dilatation operators are given by $[13,16,19,40]$

$$
D_{1}^{G}=-\sum_{\substack{i, j=1 \\ i \neq j}}^{p} n_{i j}(\sigma) \Delta_{i j}^{(1)} \quad D_{2}^{G}=-\sum_{\substack{i, j=1 \\ i \neq j}}^{p} n_{i j}(\sigma)\left\{\left(L-2 N_{c}\right) \Delta_{i j}^{(1)}+\Delta_{i j}^{(2)}\right\}
$$

where $^{5}$

$$
\begin{aligned}
& \Delta_{i j}^{(1)}=\Delta_{i j}^{+}+\Delta_{i j}^{0}+\Delta_{i j}^{-} \\
& \Delta_{i j}^{(2)}=\left(\Delta_{i j}^{+}\right)^{2}+\Delta_{i j}^{0} \Delta_{i j}^{+}+\Delta_{i j}^{+} \Delta_{i j}^{-}+\Delta_{i j}^{-} \Delta_{i j}^{+}+\Delta_{i j}^{0} \Delta_{i j}^{-}+\left(\Delta_{i j}^{-}\right)^{2} .
\end{aligned}
$$

The difference operators $\Delta_{i j}^{0}, \Delta_{i j}^{\mp}$ are defined by

$$
\begin{aligned}
& \Delta_{i j}^{0} O(\vec{l})=-\left(h\left(i, l_{i}\right)+h\left(j, l_{j}\right)\right) O(\vec{l}) \\
& \Delta_{i j}^{-} O(\vec{l})=\sqrt{h\left(i, l_{i}\right) h\left(j, l_{j}+1\right)} O\left(\ldots, l_{i}-1, \ldots, l_{j}+1, \ldots\right) \\
& \Delta_{i j}^{+} O(\vec{l})=\sqrt{h\left(i, l_{i}+1\right) h\left(j, l_{j}\right)} O\left(\ldots, l_{i}+1, \ldots, l_{j}-1, \ldots\right)
\end{aligned}
$$

where $h\left(i, l_{i}\right)$ is the box weight for spherical giants,

$$
h\left(i, l_{i}\right) \equiv N_{c}+i-l_{i}, \quad h\left(i, l_{i} \mp 1\right)-h\left(i, l_{i}\right)= \pm 1 .
$$

We find it convenient to keep $O(1)$ terms in (2.9) although $h\left(i, l_{i}\right) \sim h\left(i, l_{i} \mp 1\right)$ at large $N_{c}$. An important feature of $D_{\ell}^{G}$ is that it consists of a sum over a pair of indices $(i, j)$, and the third row/column does not show up.

We can simplify the difference operators in (2.9) by introducing

$$
\begin{aligned}
d_{i}^{-} O(\vec{l}) & =\sqrt{h\left(i, l_{i}\right)} O\left(\ldots, l_{i}-1, \ldots\right) \\
d_{i}^{+} O(\vec{l}) & =\sqrt{h\left(i, l_{i}+1\right)} O\left(\ldots, l_{i}+1, \ldots\right) \\
\hat{h}_{i} O(\vec{l}) & =h\left(i, l_{i}\right) O(\vec{l}) .
\end{aligned}
$$

These operators satisfy the relations

$$
d_{i}^{+} d_{i}^{-}=\hat{h}_{i}, \quad\left[d_{i}^{+}, d_{j}^{-}\right]=\delta_{i j}
$$

\footnotetext{
${ }^{5}$ We slightly modified the result of [19] which computed only the first term of $D_{2}$ in (2.4). We added the third term of $D_{2}$ and symmetrized $\Delta_{i j}^{(2)}$ with respect to $i \leftrightarrow j$, owing to $n_{i j}(\sigma)=n_{j i}(\sigma)$. Note that we sum over $i \neq j$ and not $i<j$.
} 
which follow from

$$
\left[d_{i}^{+}, d_{i}^{-}\right] O(\vec{l})=\left\{h\left(i, l_{i}\right)-h\left(i, l_{i}+1\right)\right\} O(\vec{l})=O(\vec{l}) .
$$

Note that

$$
\sum_{i=1}^{p}\left(N_{c}+i-\hat{h}_{i}\right) O(\vec{l})=n_{Z} O(\vec{l})
$$

We can rewrite the difference operators in (2.8) as

$$
\begin{aligned}
& \Delta_{i j}^{(1)}=-\left(d_{i}^{+}-d_{j}^{+}\right)\left(d_{i}^{-}-d_{j}^{-}\right) \\
& \Delta_{i j}^{(2)}=-\left(d_{i}^{+}-d_{j}^{+}\right)\left(1+d_{i}^{+} d_{j}^{-}+d_{j}^{+} d_{i}^{-}\right)\left(d_{i}^{-}-d_{j}^{-}\right) .
\end{aligned}
$$

If we introduce

$$
\begin{aligned}
& \mathcal{H}_{1}=\sum_{i \neq j}^{p} n_{i j}(\sigma) \mathcal{H}_{1, i j} \equiv \sum_{i \neq j}^{p} n_{i j}(\sigma)\left(d_{i}^{+}-d_{j}^{+}\right)\left(d_{i}^{-}-d_{j}^{-}\right) \\
& \mathcal{H}_{2}=\sum_{i \neq j}^{p} n_{i j}(\sigma) \mathcal{H}_{2, i j} \equiv \sum_{i \neq j}^{p} n_{i j}(\sigma)\left(d_{i}^{+}-d_{j}^{+}\right)\left(d_{i}^{+} d_{j}^{-}+d_{j}^{+} d_{i}^{-}\right)\left(d_{i}^{-}-d_{j}^{-}\right)
\end{aligned}
$$

we obtain

$$
D_{1}^{G}=-\mathcal{H}_{1}, \quad D_{2}^{G}=-\left(L-2 N_{c}\right) \mathcal{H}_{1}-\mathcal{H}_{2} .
$$

The dilatation operator $D_{\ell}^{G}$ written in terms of $\left\{d_{i}^{+}, d_{i}^{-}\right\}$can be regarded as the Hamiltonian of an effective $\mathrm{U}(p)$ theory. This is because $E_{i j} \equiv d_{i}^{+} d_{j}^{-}$satisfies the $\mathrm{GL}(p)$ commutation relations, ${ }^{6}$

$$
\left[E_{i j}, E_{k l}\right]=\delta_{j k} E_{i l}-\delta_{i l} E_{k j}
$$

We assume that $n_{i j}(\sigma)$ are general non-negative integers. Then the perturbative Hamiltonians in (2.17), (2.18) are invariant only under $\mathrm{U}(1)^{p}$.

\subsection{Commutation relations}

The one- and two-loop dilatations at $p=2$ in (2.17), (2.18) satisfy the relation

$$
\left[\mathcal{H}_{1, i j}, \mathcal{H}_{2, i j}\right]=0
$$

and the perturbative dilatation operators on the Gauss graph basis commute. This is a surprising feature because the planar dilatation operators (on the single-trace operators) at one- and two-loop do not commute [37]. ${ }^{7}$

\footnotetext{
${ }^{6}$ Roughly speaking, $E_{i j}$ is a $p \times p$ matrix whose entries are zero except at the $i$-th row, $j$-th column. The Hermitian combinations generate $\mathrm{U}(p)$.

${ }^{7}$ In general, the operator $C(g)=A+g B$ has the eigenvectors independent of $g$ when $[A, B]=0$. Conversely, if $[A, B] \neq 0$, the matrix elements of $A$ and $B$ in the eigenbasis of $C(g)$ have off-diagonal elements depending on $g$.
} 
Let us take a closer look at the situation for $p>2$. The condition $\left[D_{1}^{G}, D_{2}^{G}\right]=0$ reduces to $\left[\mathcal{H}_{1}, \mathcal{H}_{2}\right]=0$. If we impose this condition for any $\left\{n_{i j}(\sigma)\right\}$, we get

$$
\begin{aligned}
0 & =\sum_{i j, i^{\prime} j^{\prime}} n_{i j}(\sigma) n_{i^{\prime} j^{\prime}}(\sigma)\left[\mathcal{H}_{1, i j}, \mathcal{H}_{2, i^{\prime} j^{\prime}}\right] \\
& =\sum_{i j} n_{i j}(\sigma)^{2}\left[\mathcal{H}_{1, i j}, \mathcal{H}_{2, i j}\right]+\sum_{i j k} n_{i j}(\sigma) n_{i k}(\sigma)\left(\left[\mathcal{H}_{1, i j}, \mathcal{H}_{2, i k}\right]+\left[\mathcal{H}_{1, i k}, \mathcal{H}_{2, i j}\right]\right)
\end{aligned}
$$

giving us

$$
0=\left[\mathcal{H}_{1, i j}, \mathcal{H}_{2, i j}\right]=\left[\mathcal{H}_{1, i j}, \mathcal{H}_{2, i k}\right]+\left[\mathcal{H}_{1, i k}, \mathcal{H}_{2, i j}\right] .
$$

By explicit computation, one finds

$$
\left[\mathcal{H}_{1, i j}, \mathcal{H}_{2, i j}\right]=0 \quad \text { but } \quad\left[\mathcal{H}_{1, i j}, \mathcal{H}_{2, i k}\right]+\left[\mathcal{H}_{1, i k}, \mathcal{H}_{2, i j}\right] \neq 0
$$

and hence $\left[D_{1}^{G}, D_{2}^{G}\right] \neq 0$ for $p>2$. We will see in section 3 that this is a generic feature of effective $\mathrm{U}(p)$ theory Hamiltonians under some ansatz, and not due to potentially missing terms in $D_{2}^{G}$.

This trouble can be solved in the following way. In the displaced corners approximation, we truncate the Hilbert space to a fixed number of columns, then take the large $N_{c}$ limit. We obtained the Hamiltonians $D_{\ell}^{G}$ after the Hilbert space truncation, but without taking the limit. In fact, in the continuum limit we find

$$
\left[D_{1}^{G}, D_{2}^{G}\right]=0
$$

showing that taking the large $N_{c}$ limit is a necessary step.

Note that there is a caveat in the displaced corners approximation. Even if we pick up an operator in the distant region $l_{1} \gg l_{2}$, the difference $\left(l_{1}-l_{2}\right)$ keeps decreasing due to the operator mixing, until it hits the Young diagram constraints $l_{1} \geq l_{2}$. The original one-loop mixing matrix no longer takes the simple form (2.7) when two columns have comparable lengths. We expect that these boundary effects on the anomalous dimensions are negligible at the leading order of large $N_{c}$.

\subsection{Continuum limit}

We take the continuum limit following $[14,15,19]$.

We begin with the ansatz for the dilatation eigenstates,

$$
\mathcal{O}_{f}(\sigma)=\sum_{l_{1}, l_{2}, \ldots, l_{p}}^{\prime} f\left(l_{1}, l_{2}, \ldots, l_{p}\right) O^{R, r_{1}}(\sigma), \quad N_{c} \geq l_{1} \geq l_{2} \geq \cdots \geq l_{p} \geq 0, \quad \sum_{i=1}^{p} l_{i}=n_{Z}
$$

where we specify the column lengths of $r_{1}$ by $\left(l_{1}, l_{2}, \ldots, l_{p}\right)$, and $\Sigma^{\prime}$ means the sum over $\left\{l_{i}\right\}$ under the constraints shown in (2.27). The action of the operators (2.11) on $O_{f}$ can be written as

$$
\begin{aligned}
& d_{i}^{-} \mathcal{O}_{f}(\sigma) \simeq \sum_{l_{1}, l_{2}, \ldots, l_{p}}^{\prime} \sqrt{h\left(i, l_{i}\right)} f\left(\ldots, l_{i}+1, \ldots\right) O^{R, r_{1}}(\sigma) \\
& d_{i}^{+} \mathcal{O}_{f}(\sigma) \simeq \sum_{l_{1}, l_{2}, \ldots, l_{p}}^{\prime} \sqrt{h\left(i, l_{i}+1\right)} f\left(\ldots, l_{i}-1, \ldots\right) O^{R, r_{1}}(\sigma)
\end{aligned}
$$


where $\simeq$ means that we neglect potential contributions from the boundary of the summation range.

Consider the following large $N_{c}$ limit

$$
n_{Z} \sim O\left(N_{c}\right), \quad l_{1} \sim O\left(N_{c}\right), \quad l_{i} \sim O\left(\sqrt{N_{c}}\right) \quad(i=2,3, \ldots, p)
$$

which is similar to the limit discussed in [13]. We prefer the square-root scaling $l_{i} \sim O\left(\sqrt{N_{c}}\right)$ to the linear scaling $l_{i} \sim O\left(N_{c}\right)$, because the difference equations are rather trivial in the latter limit. Physically, the system (2.29) consists of one nearly maximal, and $(p-1)$ far-from maximal spherical giant gravitons. The constraint $\sum_{i} l_{i}=n_{Z}$ becomes somewhat trivial because $n_{Z} \sim l_{1}$.

We introduce the rescaled variables and functions

$$
\begin{aligned}
y_{i} & =\frac{l_{i}}{\sqrt{\alpha N_{c}}}, \quad \sqrt{\frac{N_{c}}{\alpha}} \geq y_{1} \geq y_{2} \geq \cdots \geq y_{p} \geq 0 \\
F\left(y_{1}, y_{2}, \ldots, y_{p}\right) & \equiv f\left(\frac{l_{1}}{\sqrt{\alpha N_{c}}}, \frac{l_{2}}{\sqrt{\alpha N_{c}}}, \ldots, \frac{l_{p}}{\sqrt{\alpha N_{c}}}\right) .
\end{aligned}
$$

We keep $y_{1}$ to simplify our notation, even though $y_{1}=O\left(\sqrt{N_{c}}\right) \gg 1$. It follows that

$$
\begin{aligned}
h\left(i, l_{i}\right) & =N_{c}+i-y_{i} \sqrt{\alpha N_{c}} \\
f\left(\ldots, l_{i} \pm 1, \ldots\right) & =F\left(\ldots, y_{i} \pm \frac{1}{\sqrt{\alpha N_{c}}}, \ldots\right) .
\end{aligned}
$$

In the continuum limit, the difference operators $\mathcal{H}_{1, i j}, \mathcal{H}_{2, i j}$ in $(2.17),(2.18)$ become

$$
\mathcal{H}_{1, i j} \rightarrow \mathcal{D}_{i j}, \quad \mathcal{H}_{2, i j} \rightarrow 2 N_{c} \mathcal{D}_{i j}, \quad \mathcal{D}_{i j} \equiv \frac{\alpha}{4} y_{i j}^{2}-\frac{1}{\alpha} \frac{\partial^{2}}{\partial y_{i j}^{2}}
$$

where $y_{i j}=y_{i}-y_{j}$. This suggests that the one-loop and two-loop dilatations commute in this limit,

$$
\left[D_{1}^{G}, D_{2}^{G}\right] \rightarrow 0 .
$$

The spectrum of $D_{1}^{G}$ is discussed in detail in appendix B.

\section{All-loop ansatz}

We conjecture that perturbative dilatation operators at all loops in the continuum limit (2.29) takes the form

$$
D^{G}=D_{0}+f_{c}(\lambda) \sum_{\substack{i, j=1 \\ i \neq j}}^{p} n_{i j}(\sigma) \mathcal{D}_{i j}, \quad \lambda \equiv N_{c} g_{\mathrm{YM}}^{2} .
$$

A related argument was given in [20], where they showed that the mixing of $Y$ 's at higher loops takes the same form as the one-loop mixing. 


\subsection{Constraints on higher-loop dilatations}

We expand the dilatation operator on the Gauss graph basis at weak coupling as

$$
D^{G}\left(g_{\mathrm{YM}}\right)=\sum_{\ell=0}^{\infty}\left(\frac{g_{\mathrm{YM}}}{4 \pi}\right)^{2 \ell} D_{\ell}^{G}
$$

and make the following ansatz for $D_{\ell}^{G}$ in the leading order of large $N_{c}$,

$$
D_{\ell}^{G}=\sum_{k=1}^{\ell} N_{c}^{\ell-k} x_{k} \mathcal{H}_{k}, \quad \mathcal{H}_{\ell}=\sum_{i \neq j}^{p} n_{i j}(\sigma) \mathcal{H}_{\ell, i j}
$$

where $\left\{x_{k}\right\}$ are numerical constants of $O(1)$. The first equation (3.3) means that the $\ell$-loop dilatation contains lower-loop difference operators multiplied by powers of $N_{c}$. The second equation means that $\mathcal{H}_{\ell}$ depends only on a pair of column labels $(i, j)$ coupled to $n_{i j}(\sigma)$. We impose this condition because $n_{i j}(\sigma)$ should count the number of open string modes stretching between the $i$-th and $j$-th giant graviton brane.

We further assume that

$$
\mathcal{H}_{\ell, i j} \equiv \sum_{m} \tilde{x}_{\ell m} \mathcal{P}_{\ell, m}\left(d_{i}^{\dagger}, d_{j}^{\dagger}, d_{i}, d_{j}\right), \quad \mathcal{P}_{\ell, m} \text { contains } \ell d^{\dagger} \text { 's followed by } \ell d \text { 's }
$$

where $\left\{\tilde{x}_{\ell, m}\right\}$ are numerical constants of $O(1)$, and $\mathcal{P}_{\ell, m}$ is a polynomial of the difference operators. The form of $\mathcal{P}_{\ell, m}$ originates from the perturbative dilatation operators of $\mathcal{N}=4$ SYM discussed in section 2.1. It is known that there is a correspondence between the terms of $\Delta_{i j}^{(2)}$ in (2.8) and those of $D_{2}$, according to the two-loop computation [19]. ${ }^{8}$ Since the $\ell$-loop dilatation operator $D_{\ell}$ should remove at most $\ell$ fields and add $\ell$ fields, we arrive at the ansatz of $\mathcal{P}_{\ell, m}$ in (3.4). ${ }^{9}$

Let us revisit the commutation relations in section 2.2. Now we impose

$$
\left[D^{G}\left(g_{\mathrm{YM}}\right), D^{G}\left(g_{\mathrm{YM}}^{\prime}\right)\right]=0, \quad\left(\forall g_{\mathrm{YM}}, g_{\mathrm{YM}}^{\prime}\right) \quad \Leftrightarrow \quad\left[D_{k}^{G}, D_{l}^{G}\right]=0, \quad(\forall k, l)
$$

by generalizing (2.26). This is a crucial assumption in our discussion, and should be justified in future. The equation (3.5) implies that the mixing problem can be "one-loop exact" in the sense that the eigenvectors remain unchanged at higher loop orders. ${ }^{10}$

The first equation of (2.24) is generalized to

$$
\left[\mathcal{H}_{\ell, i j}, \mathcal{H}_{\ell^{\prime}, i j}\right]=0 \quad\left(\forall \ell, \ell^{\prime}\right) .
$$

It is straightforward to enumerate all possible solutions of (3.6), or equivalently the general form of $\mathcal{P}_{\ell, m}$, with the help of Mathematica.

\footnotetext{
${ }^{8}$ For example, $\left(d_{i}^{\dagger}\right)^{2} d_{j}^{2}$ removes two boxes from the $j$-th column and add two boxes to the $i$-th column. This term comes from $\left(\Delta_{i j}^{+}\right)^{2}$ which roughly corresponds to $\operatorname{Tr}(Z Z W \check{Z} \check{Z} \check{W})$.

${ }^{9}$ We can also explain the powers of $N_{c}$ in (3.3) from the fact that $D_{\ell}$ removes $\ell$ fields and adds $\ell$ fields.

${ }^{10}$ The author thanks an anonymous referee of JHEP for correcting mistakes in the previous version and emphasizing this point.
} 
It turns out that at $\ell$-loop, there are $(\ell+1)$ independent solutions of the equation (3.6). At one-loop, there are two solutions

$$
\begin{aligned}
& \mathcal{P}_{i j, 10}=d_{i}^{\dagger} d_{j}+d_{j}^{\dagger} d_{i} \equiv \mathcal{J}_{i j} \\
& \mathcal{P}_{i j, 11}=d_{i j}^{\dagger} d_{i j} \quad=\mathcal{H}_{1, i j}
\end{aligned}
$$

where $\mathcal{H}_{1, i j}$ is given in $(2.17)$ and

$$
d_{i j}^{\dagger}=d_{i}^{\dagger}-d_{j}^{\dagger}, \quad d_{i j}=d_{i}-d_{j} .
$$

We also define

$$
\mathcal{I}_{i j} \equiv d_{i}^{\dagger} d_{i}+d_{j}^{\dagger} d_{j}=d_{i j}^{\dagger} d_{i j}-\mathcal{J}_{i j}
$$

which satisfies

$$
\left[\mathcal{I}_{i j}, \mathcal{J}_{i j}\right]=0
$$

At two-loop, there are three solutions,

$$
\begin{aligned}
& \mathcal{P}_{i j, 20}=\left(d_{i}^{\dagger}\right)^{2}\left(d_{j}\right)^{2}+2 d_{i}^{\dagger} d_{j}^{\dagger} d_{i} d_{j}+\left(d_{j}^{\dagger}\right)^{2}\left(d_{i}\right)^{2}=\mathcal{J}_{i j}^{(2)} \\
& \mathcal{P}_{i j, 21}=d_{i j}^{\dagger} \mathcal{J}_{i j} d_{i j} \quad=\mathcal{H}_{2, i j} \\
& \mathcal{P}_{i j, 22}=\left(d_{i j}^{\dagger}\right)^{2}\left(d_{i j}\right)^{2} \quad=: \mathcal{H}_{1, i j}^{2}:
\end{aligned}
$$

where $\mathcal{H}_{2, i j}$ is given in $(2.18)$ and

$$
\mathcal{J}_{i j}^{(n)} \equiv:\left(\mathcal{J}_{i j}\right)^{n}:=\sum_{m=0}^{n}\left(\begin{array}{c}
n \\
m
\end{array}\right)\left(d_{i}^{\dagger}\right)^{m}\left(d_{j}^{\dagger}\right)^{n-m}\left(d_{i}\right)^{n-m}\left(d_{j}\right)^{m} .
$$

At higher loops, we find that all solutions at $\ell$-loop can be written in the form

$$
\mathcal{Q}_{a b}=\mathcal{Q}_{a b, i j} \equiv\left(d_{i j}^{\dagger}\right)^{a} \mathcal{J}_{i j}^{(b)}\left(d_{i j}\right)^{a}, \quad(a=\ell-b=0,1, \ldots, \ell)
$$

We checked that no more solutions exist up to four-loop. The two-parameter family of difference operators (3.16) mutually commute,

$$
\left[\mathcal{Q}_{a b}, \mathcal{Q}_{a^{\prime} b^{\prime}}\right]=0 \quad\left(\forall a, b, a^{\prime}, b^{\prime}\right)
$$

which follows from (3.10) and (3.11). Our ansatz for the $\ell$-loop dilatation in (3.3) becomes

$$
\mathcal{H}_{\ell, i j}=\sum_{m=0}^{\ell} \tilde{x}_{\ell m} \mathcal{Q}_{\ell-m, m} .
$$

The second equation of (2.24) generalized to higher loops reads

$$
\left[\mathcal{H}_{\ell, i j}, \mathcal{H}_{\ell^{\prime}, i k}\right]+\left[\mathcal{H}_{\ell, i k}, \mathcal{H}_{\ell^{\prime}, i j}\right]=0 \quad\left(\forall \ell, \ell^{\prime}\right) .
$$

Some of $\mathcal{Q}_{a b, i j}$ in (3.16) up to two loops satisfy these conditions. At three-loops, no linear combinations of $\left(\mathcal{Q}_{3,0}, \mathcal{Q}_{2,1}, \mathcal{Q}_{1,2}, \mathcal{Q}_{0,3}\right)$ satisfy (3.19) against the two-loop dilatation. Thus, we should trust the discrete form of our all-loop ansatz (3.3) only at $p=2$. 
This conclusion is not surprising. The effective Hamiltonian $\mathcal{H}_{\ell, i j}$ is a linear combination of $\mathcal{Q}_{a b, i j}$ in (3.16), which are polynomials of $\mathcal{J}_{i j}$. However, $\mathcal{J}_{12}$ and $\mathcal{J}_{23}$ as the $\mathfrak{s u}(p)$ generators (2.20) do not commute:

$$
\left[\mathcal{J}_{12}, \mathcal{J}_{23}\right] \sim\left[\left(\begin{array}{lll}
0 & 1 & 0 \\
1 & 0 & 0 \\
0 & 0 & 0
\end{array}\right),\left(\begin{array}{lll}
0 & 0 & 0 \\
0 & 0 & 1 \\
0 & 1 & 0
\end{array}\right)\right]=\left(\begin{array}{ccc}
0 & 0 & 1 \\
0 & 0 & 0 \\
-1 & 0 & 0
\end{array}\right) \neq 0
$$

which makes it hard to solve (3.19). See also the discussion in section 2.2.

We can determine the numerical coefficients in (3.3), (3.18) up to two loops. By comparing $\mathcal{Q}_{a b}$ with the perturbative results (2.19), we find

$$
\begin{aligned}
& D_{1}^{G}=-\sum_{i \neq j} n_{i j}(\sigma)\left\{0 \cdot \mathcal{Q}_{0,1}+1 \cdot \mathcal{Q}_{1,0}\right\} \\
& D_{2}^{G}=-\sum_{i \neq j} n_{i j}(\sigma)\left\{0 \cdot \mathcal{Q}_{0,1}+\left(L-2 N_{c}\right) \mathcal{Q}_{1,0}+0 \cdot \mathcal{Q}_{0,2}+1 \cdot \mathcal{Q}_{1,1}+0 \cdot \mathcal{Q}_{2,0}\right\}
\end{aligned}
$$

implying that most coefficients vanish in $\mathcal{N}=4 \mathrm{SYM}$. This result is also consistent with our assumption in (3.3) that $\left\{\tilde{x}_{\ell m}\right\}$ are numerical constants of $O(1)$.

\subsection{Continuum limit at higher loops}

By combining (3.3) and (3.18), we obtain a conjecture of the $\ell$-loop dilatation operator,

$$
D_{\ell}^{G}=\sum_{i \neq j}^{p} n_{i j}(\sigma) \sum_{k=1}^{\ell} \sum_{m=0}^{k} N_{c}^{\ell-k} C_{k, m} \mathcal{Q}_{k-m, m}, \quad C_{k, m}=x_{k} \tilde{x}_{k m}=O\left(N_{c}^{0}\right) .
$$

The terms with $k<\ell$ are part of the dilatation at lower loop orders, combined with powers of $N_{c}$.

Let us take the continuum limit (2.29). The commuting operators $\mathcal{Q}_{a b}$ in (3.16) scale as ${ }^{11}$

$$
\mathcal{Q}_{\ell-m, m} \sim N_{c}^{m}
$$

and in particular

$$
\begin{aligned}
& \mathcal{Q}_{0, m}=\left(2 N_{c}\right)^{m}+O\left(N_{c}^{m-1 / 2}\right) \\
& \mathcal{Q}_{1, m}=\left(2 N_{c}\right)^{m} \mathcal{D}_{i j}+O\left(N_{c}^{m-1 / 2}\right) \\
& \mathcal{Q}_{2, m}=\left(2 N_{c}\right)^{m}\left(\frac{\alpha^{2}}{16} y_{i j}^{4}-\frac{y_{i j}^{2}}{2} \frac{\partial^{2}}{\partial y_{i j}^{2}}+\frac{1}{\alpha^{2}} \frac{\partial^{4}}{\partial y_{i j}^{4}}\right)+O\left(N_{c}^{m-1 / 2}\right) \\
& \mathcal{Q}_{3, m}=\left(2 N_{c}\right)^{m}\left(\frac{\alpha^{3}}{64} y_{i j}^{6}-\frac{3 y_{i j}^{4}}{16} \frac{\partial^{2}}{\partial y_{i j}^{2}}+\frac{3 y_{i j}^{2}}{4} \frac{\partial^{4}}{\partial y_{i j}^{4}}-\frac{1}{\alpha^{2}} \frac{\partial^{6}}{\partial y_{i j}^{6}}\right)+O\left(N_{c}^{m-1 / 2}\right)
\end{aligned}
$$

where $\mathcal{D}_{i j}$ is given in (2.33). From this observation, we can refine (3.23) as

$$
\mathcal{Q}_{\ell, m}=\left(2 N_{c}\right)^{m}: \mathcal{D}_{i j}^{\ell}:+O\left(N_{c}^{m-1 / 2}\right)
$$

where : $\mathcal{D}_{i j}^{\ell}$ : means that the derivative $\left(\partial / \partial y_{i j}\right)$ should not hit $y_{i j}$ in the subsequent $\mathcal{D}_{i j}$ 's.

\footnotetext{
${ }^{11}$ Before the continuum limit, $\mathcal{Q}_{a b}$ scales as $N_{c}^{a+b}$.
} 
Then, our conjectured dilatation operator (3.22) becomes

$$
D_{\ell}^{G}=\sum_{i \neq j}^{p} n_{i j}(\sigma) \sum_{k=1}^{\ell} \sum_{m=0}^{k} N_{c}^{\ell-k+m}\left(2^{m} C_{k, m}\right): \mathcal{D}_{i j}^{k-m}: .
$$

We find that the terms $m=k$ are leading at large $N_{c}$. However, the perturbative data (3.21) shows that $C_{k, k}=0$. The terms $m=k-1$ gives the first non-vanishing term, which is proportional to the one-loop result $D_{1}^{G}$. The terms $m \leq k-2$ are negligible as long as $C_{k, m}=O\left(N_{c}^{0}\right)$.

Given (3.26) we can formally sum up the perturbation series,

$$
D^{G}=D_{0}+\sum_{\ell=1}^{\infty} g_{\mathrm{YM}}^{2 \ell} D_{\ell}^{G} \rightarrow D_{0}+N_{c}^{-1} f_{c}(\lambda) \sum_{i \neq j} n_{i j}(\sigma) \mathcal{D}_{i j}
$$

where $\lambda=N_{c} g_{\mathrm{YM}}^{2}$ is the 't Hooft coupling and

$$
f_{c}(\lambda)=\sum_{\ell=1}^{\infty} \lambda^{\ell} \sum_{k=1}^{\ell} 2^{k-1} C_{k, k-1} .
$$

This is the result quoted in (3.1). According to appendix B.1, the operator $\mathcal{D} \equiv$ $\sum n_{i j}(\sigma) \mathcal{D}_{i j}$ has the eigenvalues (B.14). Thus

$$
D^{G}-D_{0}=N_{c}^{-1} f_{c}(\lambda)\left\{n_{Y}+2 \sum_{a=1}^{p-1}\left(2 m_{a}+1\right) \tilde{\lambda}_{a}\left(\left\{n_{i j}\right\}\right)\right\}
$$

where $\lambda_{a}$ depends on $n_{i j}(\sigma)$ and has the same order as $n_{Y}$. We will see in appendix B.2 that the non-negative integers $m_{a}$ should be bounded from above, and at most $O\left(N_{c}\right)$. Neglecting $O(1)$ quantities, the equation (3.29) becomes

$$
D^{G} \rightarrow D_{0}+\tilde{f}(\lambda) \sum_{a=1}^{p-1} \frac{m_{a}}{N_{c}} \tilde{\lambda}_{a}\left(\left\{n_{i j}\right\}\right)
$$

with $\tilde{f}(\lambda)=4 f_{c}(\lambda)$. When $p=2$, we obtain

$$
\begin{gathered}
D^{G}=L+\tilde{f}(\lambda) \frac{m}{N_{c}} n_{12}(\sigma), \quad \tilde{f}(\lambda)=\frac{\lambda}{2 \pi^{2}}+O\left(\lambda^{2}\right) \\
n_{12}(\sigma) \in \mathbb{Z}_{\geq 0}, \quad m=1,2, \ldots,\left\lceil N_{c}-\frac{n_{Z}}{2}+1\right\rceil
\end{gathered}
$$

where we used (2.1) and (B.37).

The factor $m_{a} / N_{c}$ in (3.30) has the spacing of order $1 / N_{c}$, which becomes continuous at large $N_{c}$. If $f_{c}(\lambda)$ remains non-zero at $\lambda \gg 1$, then the above ansatz should describe (semi)classical motion of the system with D-branes and strings. Importantly, this excitation spectrum should be gapless.

\section{Strong coupling}

We want to reproduce the dilatation spectrum (3.30) at strong coupling. Since the energy of excited states is continuously connected to the BPS state, we take a classical D3-brane action and study the solution around the BPS giant graviton. 


\subsection{D3-brane action}

The action for a single D3-brane is given by

$$
S=S_{\mathrm{DBI}}+S_{\mathrm{CS}}=-T_{3} \int_{\Sigma_{4}} d^{4} \xi e^{-\varphi} \sqrt{-\operatorname{det}\left(G_{a b}+B_{a b}+2 \pi \alpha^{\prime} F_{a b}\right)}+T_{3} \int_{\Sigma_{4}} C^{(4)}
$$

where $\Sigma_{4}$ is the worldvolume, $G_{a b}=g_{\mu \nu} \partial_{a} X^{\mu} \partial_{b} X^{\nu}$ is the induced metric. We consider a D3-brane wrapping $\mathrm{S}^{3}$ inside $\mathrm{AdS}_{5} \times \mathrm{S}^{5}$,

$$
\text { D3 }: \Sigma_{4} \rightarrow \mathbb{R} \times \mathrm{S}^{3} \subset \mathrm{AdS}_{5} \times \mathrm{S}^{5}
$$

which includes the spherical giant graviton. There is no $B_{a b}$ and $F_{a b}$ in the background, and the dilaton is constant, $e^{\varphi}=g_{s}$. The constant $T_{3}$ is given by [4]

$$
T_{3}=\frac{2 \pi}{g_{s}\left(4 \pi^{2} \alpha^{\prime}\right)^{2}}=\frac{N_{c}}{R^{4} \Omega_{3}}
$$

where $R$ is the radius of $\operatorname{AdS}_{5} \times \mathrm{S}^{5}$ and $\Omega_{3}=2 \pi^{2}$ is the volume of $\mathrm{S}^{3}$ with the unit radius. Our notation for the $\mathrm{AdS}_{5} \times \mathrm{S}^{5}$ geometry is explained in appendix D.1.

The induced metric is written as

$$
\begin{aligned}
G_{a b}= & R^{2}\left\{-\partial_{a} t \partial_{b} t+\frac{\partial_{a} \rho \partial_{b} \rho}{4(\rho-1) \rho^{2}}+\frac{(\rho-1) \partial_{a} \phi \partial_{b} \phi}{\rho}\right. \\
& \left.+\frac{\partial_{a} \eta \partial_{b} \eta+\cos ^{2} \eta \partial_{a} \theta_{1} \partial_{b} \theta_{1}+\sin ^{2} \eta \partial_{a} \theta_{2} \partial_{b} \theta_{2}}{\rho}\right\} .
\end{aligned}
$$

We choose the static gauge

$$
t=\xi^{0}, \quad \theta_{1}=\xi^{1}, \quad \theta_{2}=\xi^{2}, \quad \eta=\xi^{3}
$$

and assume the ansatz

$$
\rho=\rho(t, \eta), \quad \phi=\phi(t, \eta) .
$$

The D3-brane action effectively becomes two-dimensional, ${ }^{12}$

$$
S \equiv \int_{\mathbb{R} \times S^{3}} d^{4} \xi \mathcal{L}=\frac{N_{c}}{g_{s} \Omega_{3}} \int d^{4} \xi\left(-\sqrt{-\operatorname{det} G}+\delta_{a 0} \sin \eta \cos \eta \frac{\left(\partial_{a} \phi\right)}{\rho^{2}}\right) .
$$

The conserved charges can be computed in the standard way,

$$
\begin{aligned}
& J=\int_{0}^{\pi / 2} d \eta \mathrm{j}^{0}(t, \eta)=\int d \eta \frac{\delta S}{\delta \partial_{0} \phi} \\
& E=\int_{0}^{\pi / 2} d \eta \mathrm{h}(t, \eta)=\int d \eta\left\{\sum_{X=\rho, \phi} \partial_{a} X \frac{\delta S}{\delta \partial_{a} X}-\mathcal{L}\right\} .
\end{aligned}
$$

\footnotetext{
${ }^{12}$ We chose the CS coupling so that the ground state satisfies $E=J$ including the sign, which can be flipped by $\phi \rightarrow-\phi$. The physical brane tension is proportional to $N_{c} / g_{s}$.
} 


\subsection{Classical solutions}

We study the effective two-dimensional action (4.7) around the spherical giant graviton solution as follows. We assume the static gauge, introduce the deformation parameter $\epsilon$, and solve the equations of motion (EoM) as a formal series of small $\epsilon$. The linearized EoM are given by a set of homogeneous partial differential equations, whose coefficients may depend on $\eta$. We remove the $\eta$ dependence by the separation of variables for the deformed degrees of freedom.

This procedure looks similar to the analysis of one-loop fluctuation [27, 29]. Generally, however, not all off-shell fluctuations become the deformed solutions of the classical equations of motion.

Other deformations of the spherical giant graviton solution might be possible if the ansatz and the gauge choice are generalized. ${ }^{13}$ One can try to study the deformation in the AdS directions, and to search for the solutions with non-zero $U(1)$ field strength or other components of $\mathrm{SO}(6)$ angular momenta.

\subsubsection{Ground state}

The ansatz for the ground (or BPS) state of a spherical giant graviton is [4]

$$
\rho=\text { constant }, \quad \phi=t .
$$

The energy as a function of $\rho$ has a local minimum at $\rho=N_{c} /\left(g_{s} J\right)$, and the minimum value is

$$
E=J
$$

\subsubsection{Excited states}

We are interested in the non-BPS states which are continuously connected to the BPS state. Let us generalize the ansatz by expanding around the ground state solution as

$$
\rho=\frac{1}{j}+\epsilon \rho_{1}(t, \eta), \quad \phi=t+\epsilon \phi_{1}(t, \eta), \quad j \equiv \frac{g_{s} J}{N_{c}} .
$$

We consider the EoM for three cases, $j=0,0<j<1$ and $j=1$. No non-trivial solutions are found for the cases $j=0,1$, as discussed in appendix D.3. When $0<j<1$, the EoM for $\phi$ and $\rho$ take the form

$$
\begin{aligned}
\frac{j^{2} \partial_{t} \rho_{1}}{j-1}+\partial_{t}^{2} \phi_{1} & =\partial_{\eta}^{2} \phi_{1}+2 \cot (2 \eta) \partial_{\eta} \phi_{1} \\
-\frac{4(j-1) \partial_{t} \phi_{1}}{j^{2}}+\partial_{t}^{2} \rho_{1} & =\partial_{\eta}^{2} \rho_{1}+2 \cot (2 \eta) \partial_{\eta} \rho_{1}
\end{aligned}
$$

which can be solved by separation of variables. The r.h.s. of (4.12), (4.13) are identical to the Laplacian on $\mathrm{S}^{3}$, whose normalizable solutions are given by the spherical harmonics (D.6). Since our $\phi_{1}$ and $\rho_{1}$ are independent of $\theta_{1}, \theta_{2}$, we set

$$
\rho_{1}(t, \eta)=\tilde{\rho}_{1}(t) \Phi_{k, 0,0}(\eta), \quad \phi_{1}(t, \eta)=\tilde{\phi}_{1}(t) \Phi_{k, 0,0}(\eta) .
$$

\footnotetext{
${ }^{13}$ The gauge choice may change the CS term.
} 
Then the general solution of the equations (4.12), (4.13) is given by

$$
\begin{aligned}
\tilde{\rho}_{1}(t)= & \frac{1}{j^{2}}\left[c_{(1)}((k+2) \cos (k t)+k \cos ((k+2) t))+c_{(2)} \cos t \sin ((k+1) t)\right. \\
& \left.+c_{(3)}(k \sin ((k+2) t)-(k+2) \sin (k t))+c_{(4)} \sin t \sin ((k+1) t)\right] \\
\tilde{\phi}_{1}(t)= & \frac{1}{2(j-1)}\left[c_{(1)}((k+2) \sin (k t)-k \sin ((k+2) t))-c_{(2)} \sin t \sin ((k+1) t)\right. \\
& \left.+c_{(3)}((k+2) \cos (k t)+k \cos ((k+2) t))+c_{(4)} \cos t \sin ((k+1) t)\right]
\end{aligned}
$$

where $c_{(i)}(i=1,2,3,4)$ are integration constants.

Let us compute the corrections to the conserved charges from (4.14),

$$
E=\sum_{n=0} \epsilon^{n} E_{(n)}=\sum_{n=0} \epsilon^{n} \int_{0}^{\pi / 2} d \eta \mathrm{h}_{(n)}, \quad J=\sum_{n=0} \epsilon^{n} J_{(n)}=\sum_{n=0} \epsilon^{n} \int_{0}^{\pi / 2} d \eta \mathrm{j}_{(n)}^{0} .
$$

It follows that

$$
\mathrm{h}_{(1)}=\mathrm{j}_{(1)}^{0}=\frac{N_{c}}{g_{s} \Omega_{3}} \sin \eta \cos \eta \Phi_{k, 0,0}(\eta)\left\{(1-j) \partial_{t} \tilde{\phi}_{1}-j^{2} \tilde{\rho}_{1}(t)\right\} .
$$

Only the $k=0$ term remains non-zero after the integration over $\mathrm{S}^{3}$ owing to the orthogonality (D.9). We remove the first-order correction to the conserved charges by setting

$$
E_{(1)}=J_{(1)} \propto\left(4 c_{(1)}+c_{(4)}\right)=0 .
$$

The difference $(E-J)$ is non-zero at the second order in the $\epsilon$ expansion,

$$
\begin{aligned}
\mathrm{h}_{(2)}-j_{(2)}^{0}= & \frac{N_{c}}{g_{s} \Omega_{3}} \frac{\sin 2 \eta}{16(1-j)}\left\{\Phi_{k, 0,0}(\eta)^{2}\left(j^{4}\left(\partial_{t} \tilde{\rho}_{1}\right)^{2}+4(j-1)^{2}\left(\partial_{t} \tilde{\phi}_{1}\right)^{2}\right)\right. \\
& \left.-\left(\partial_{\eta} \Phi_{k, 0,0}\right)^{2}\left(j^{4} \tilde{\rho}_{1}^{2}+4(j-1)^{2} \tilde{\phi}_{1}^{2}\right)\right\} \\
\simeq & \frac{N_{c}}{g_{s} \Omega_{3}} \frac{\sin 2 \eta \Phi_{k, 0,0}(\eta)^{2}}{16(1-j)}\left\{\left(j^{4}\left(\partial_{t} \tilde{\rho}_{1}\right)^{2}+4(j-1)^{2}\left(\partial_{t} \tilde{\phi}_{1}\right)^{2}\right)\right. \\
& \left.-k(k+2)\left(j^{4} \tilde{\rho}_{1}^{2}+4(j-1)^{2} \tilde{\phi}_{1}^{2}\right)\right\}
\end{aligned}
$$

where $\simeq$ denotes the equality after the integration over $S^{3}$ coming from (D.10).

Here we encounter apparent inconsistency. The corrections to the conserved charges (4.20) may depend on $t$ even after the integration over $\mathrm{S}^{3}$. This is partly because our ansatz (4.11) solves the EoM only at $O(\epsilon)$ whereas the corrections are $O\left(\epsilon^{2}\right)$. This explanation is not entirely correct because the solutions at $O\left(\epsilon^{2}\right)$ do not seem to change $E-J$ at $O\left(\epsilon^{2}\right)$. Fortunately we can remove the $t$ dependence either by adjusting the constants $\left\{c_{i}\right\}$, or by setting $k=0$.

The general $k>0$ solutions are given by

$$
\begin{aligned}
& \rho_{1}(t, \eta)=\frac{c_{k}(k+1)}{j^{2}(k+2)} \sin ((k+2) t) \Phi_{k, 0,0}(\eta) \\
& \phi_{1}(t, \eta)=\frac{c_{k}(k+1)}{2(j-1)(k+2)} \cos ((k+2) t) \Phi_{k, 0,0}(\eta)
\end{aligned}
$$


which has the dispersion relation

$$
E-J=\frac{N_{c}}{g_{s}} \frac{\epsilon^{2} c_{k}^{2}(k+1)^{2}}{8(1-j)(k+2)} .
$$

Here $k$ should be a positive even integer as in appendix D.2, and $c_{k}$ should remain finite as $k \gg 1$ in order to keep (4.21) finite. The general $k=0$ solution is

$$
\rho_{1}(t, \eta)=\frac{c_{(2)} \sin (2 t)-c_{(4)} \cos (2 t)}{2 \sqrt{2} \pi j^{2}}, \quad \phi_{1}(t, \eta)=\frac{\left(4 c_{(3)}-c_{(2)}\right)+c_{(2)} \cos (2 t)+c_{(4)} \sin (2 t)}{4 \sqrt{2} \pi(j-1)}
$$

with the dispersion relation

$$
E-J=\frac{N_{c}}{g_{s}} \frac{\epsilon^{2}\left(c_{(2)}^{2}+c_{(4)}^{2}\right)}{32 \pi^{2}(1-j)} .
$$

In (4.23), the parameter $c_{(3)}$ is redundant because it just shifts the origin of $\phi$. Also, the terms proportional to $c_{(2)}$ coincide with the $k=0$ case of the previous solution (4.21).

The profile of those with KK modes is depicted in figure 1, and the profile of the oscillating giant gravitons without KK modes is in figure 2. The former solutions expand and shrink over the area of $O\left(\epsilon^{2}\right)$. The latter solutions are point-like and oscillate around the BPS configuration.

\subsubsection{Excited states at higher orders}

We can take a linear combination of the solutions at $O(\epsilon)$ and proceed to higher orders,

$$
\begin{aligned}
& \rho=\frac{1}{j}+\epsilon\left\{\frac{C_{0} \cos (2 t)}{2 \sqrt{2} \pi j^{2}}-\sum_{k} \frac{c_{k}(1+k) \sin ((k+2) t) \Phi_{k, 0,0}(\eta)}{j^{2}(k+2)}\right\}+\sum_{n=2} \epsilon^{n} \rho_{n}(t, \eta) \\
& \phi=t+\epsilon\left\{\frac{C_{0} \sin (2 t)}{4 \sqrt{2} \pi(1-j)}-\sum_{k} \frac{c_{k}(1+k) \cos ((k+2) t) \Phi_{k, 0,0}(\eta)}{2(1-j)(k+2)}\right\}+\sum_{n=2} \epsilon^{n} \phi_{n}(t, \eta) .
\end{aligned}
$$

When $\partial_{\eta} \rho=\partial_{\eta} \phi=0$, or equivalently if $c_{k}=0$ for $k>0$, we could solve EoM at higher orders of $\epsilon$. In this case, $\phi(t)$ is fixed by the angular momentum (4.8),

$$
j=\frac{1}{\rho^{2}}+\frac{(\rho-1) R \dot{\phi}}{\rho^{5 / 2}}\left(\kappa^{2}-\frac{R^{2} \dot{\rho}^{2}}{4(\rho-1) \rho^{2}}-\frac{(\rho-1) R^{2} \dot{\phi}^{2}}{\rho}\right)^{-1 / 2} .
$$

We can solve the EoM for $\rho(t)$ as

$$
\begin{aligned}
\rho= & \frac{1}{j}+\epsilon\{\mathrm{c} \cos (2 t)+\mathrm{d} \sin (2 t)\}+\epsilon^{2} \frac{j(2 j-3)\left(\mathrm{c}^{2}+\mathrm{d}^{2}\right)}{2(j-1)} \\
& -\epsilon^{3} \frac{3 j^{2}\left(\mathrm{c}^{2}+\mathrm{d}^{2}\right)}{4(j-1)}\{(\mathrm{d}+4 \mathrm{c} t) \sin (2 t)+(\mathrm{c}-4 \mathrm{~d} t) \cos (2 t)\} \\
& -\epsilon^{4} \frac{j^{3}\left(\mathrm{c}^{2}+\mathrm{d}^{2}\right)}{8(j-1)^{3}}\left\{(2 j(j(4 j-9)+6)-3)\left(\mathrm{c}^{2}+\mathrm{d}^{2}\right)\right. \\
& \left.+2(j-1)^{2}\left(\left(\mathrm{~d}^{2}-\mathrm{c}^{2}\right) \cos (4 t)-2 \mathrm{~cd} \sin (4 t)\right)\right\}+O\left(\epsilon^{5}\right) .
\end{aligned}
$$



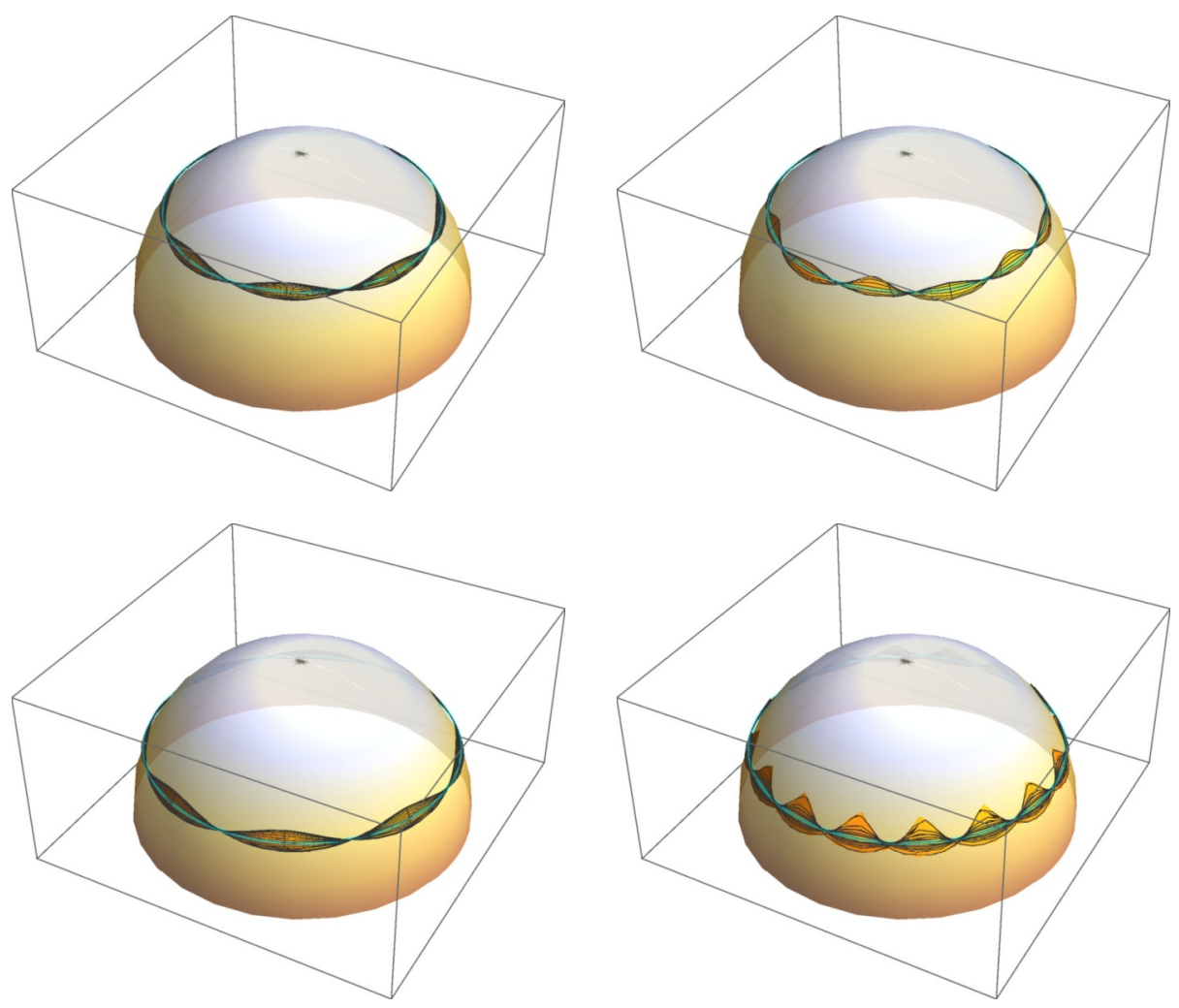

Figure 1. The trajectory of oscillating giant gravitons with KK modes. The green lines denote the BPS ground states. We set $(j, k)=(0.4,2)$ for top left, $(j, k)=(0,4,4)$ for top right, $(j, k)=(0.2,2)$ for bottom left, $(j, k)=(0,2,6)$ for bottom right. Other parameters are $R=1, \epsilon=0.15, c_{k}=1$.
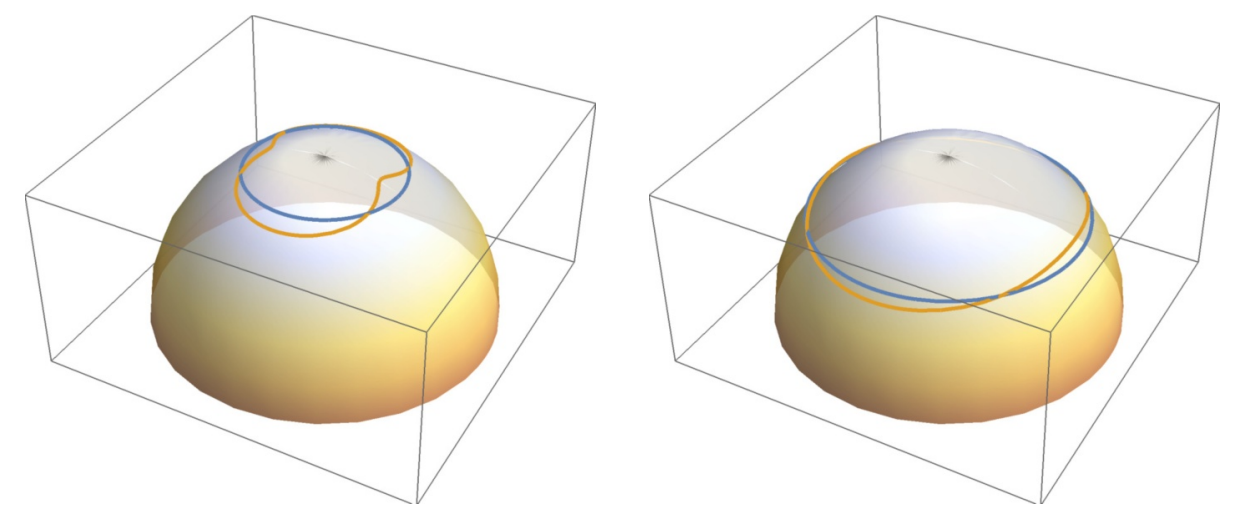

Figure 2. Giant gravitons at the vacuum (blue) and excited (orange) states. We put $j=0.8$ for the left and $j=0.4$ for the right figure. Other parameters are $R=1, \epsilon=0.05, \mathrm{c}=1, \mathrm{~d}=0$.

The parameters (c,d) are arbitrary constants of $O(1)$ which may depend on $\epsilon$. The energy of an oscillating D3 brane is

$$
E=\frac{N_{c}}{g_{s}}\left\{j+\epsilon^{2} \frac{\left(\mathrm{c}^{2}+\mathrm{d}^{2}\right) j^{4}}{2(1-j)}-\epsilon^{4} \frac{\left(\mathrm{c}^{2}+\mathrm{d}^{2}\right)^{2} j^{6}(7(j-3) j+15)}{8(1-j)^{3}}+O\left(\epsilon^{6}\right)\right\}
$$


The solution (4.27) contains secular terms like

$($ polynomial of $t) \times \cos (2 m t), \quad($ polynomial of $t) \times \sin (2 m t), \quad(m \in \mathbb{Z})$.

We should renormalize the frequencies in order to keep $\rho(t)$ finite at large $t$.

When the first-order solution has KK modes, namely if $c_{k} \neq 0$, we do not find higherorder classical solutions. One possible interpretation is that the solutions with non-trivial KK modes on $\mathrm{S}^{3}$ are not purely classical, and hence they cannot produce $E-J=O\left(N_{c}\right)>$ 0 . If we think of $\epsilon \gtrsim \lambda / N_{c} \sim g_{s}$, then the higher-order corrections are mixed up with $g_{s}$ corrections.

\section{Comments on AdS/CFT}

We look for the strong coupling counterpart of the all-loop ansatz (3.30)

$$
\Delta-J=\frac{\tilde{f}(\lambda)}{N_{c}} \sum_{\alpha=1}^{p-1} m_{\alpha} \lambda_{\alpha}\left(\left\{n_{i j}\right\}\right)
$$

where $\alpha$ labels the eigenvalues of the $(p-1)$ coupled oscillators, and $n_{i j}(\sigma)$ is a non-negative integer satisfying

$$
n_{i j}(\sigma)=n_{i \rightarrow j}(\sigma)+n_{j \rightarrow i}(\sigma), \quad \sum_{j=1}^{p} n_{i \rightarrow j}=\sum_{j=1}^{p} n_{j \rightarrow i}, \quad n_{Y}=\sum_{i=1}^{p} \sum_{j=1}^{p} n_{i \rightarrow j}(\sigma) .
$$

The second equation is the Gauss law constraints (A.13), which suggests that $n_{i j}(\sigma)$ is the number of open strings stretching between the $i$-th and $j$-th branes.

At $p=2$, the ansatz (5.1) becomes

$$
\Delta-J=\frac{\tilde{f}(\lambda)}{N_{c}} m n_{12}, \quad n_{12}(\sigma) \in \mathbb{Z}_{\geq 0}, \quad m=1,2, \ldots,\left\lceil N_{c}-\frac{n_{Z}}{2}+1\right\rceil .
$$

\subsection{Comparison with the oscillating D3-brane}

In section 4, we found that there are two types of classical D-brane motion around the BPS configuration, whose energies are given by

$$
E-J= \begin{cases}\frac{N_{c}}{g_{s}}\left[\epsilon^{2} \frac{\left(\mathrm{c}^{2}+\mathrm{d}^{2}\right) j^{4}}{2(1-j)}-\epsilon^{4} \frac{\left(\mathrm{c}^{2}+\mathrm{d}^{2}\right)^{2} j^{6}(7(j-3) j+15)}{8(1-j)^{3}}+O\left(\epsilon^{6}\right)\right] & (k=0) \\ \frac{N_{c}}{g_{s}}\left[\frac{\epsilon^{2} c_{k}^{2}(k+1)^{2}}{8(1-j)(k+2)}+O\left(\epsilon^{4}\right)\right] & (k \geq 2) .\end{cases}
$$

The first solution can be easily extended to higher orders of $\epsilon$, whereas the second solution cannot be extended to the next order by means of the simple separation of variables.

We argue that the oscillating D-brane should correspond to the harmonic oscillator of the effective $\mathrm{U}(p)$ theory. More explicitly, we relate the energy of oscillating D-brane (5.4) at large $k$ and the all-loop ansatz (5.3) at $p=2$ and large $m$,

$$
E-J \simeq \frac{N_{c}^{2} \epsilon^{2}}{\lambda} \frac{\pi c_{k}^{2}}{2(1-j)} k \quad \leftrightarrow \quad \Delta-J=\frac{\tilde{f}(\lambda)}{N_{c}} n_{12}(\sigma) m .
$$




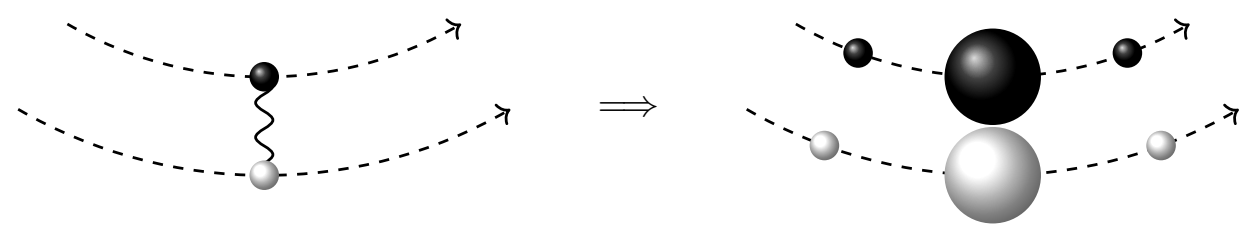

Figure 3. (Left) Open string stretching between two D3-branes as a probe. (Right) D3-branes start expanding and shrinking.

where we used $\lambda \equiv R^{4} / \alpha^{2}=4 \pi g_{s} N_{c}$. We regard $\epsilon$ as the quantity of $O\left(1 / N_{c}\right)$, which is an effect of the fundamental strings moving around the D3-brane. Then $k$ should be less than $O\left(\epsilon^{-1 / 2}\right)$ to keep the corrections to $(E-J)$ small. This bound corresponds to the fact that the mode number $m$ is bounded from above at $O\left(N_{c}\right)$.

Let us present several lines of reasoning behind this identification.

Firstly, both dispersion relations are gapless, and one can excite the BPS state by supplying an arbitrarily small amount of energy.

Secondly, let us recall the AdS/CFT correspondence for the half-BPS states. At weak coupling, the Young diagrams with different shapes start mixing at one-loop. The column length of a Young diagram can be interpreted as the radial direction of droplet patterns in the LLM plane [3]. This interpretation shows that the D-brane itself should oscillate.

Thirdly, we cannot deform the $j=1$ solution, i.e. the maximal giant graviton. This corresponds to the fact that one cannot attach a box representing $Y$ to the Gauss graph operator $\mathcal{O}^{R, r}(\sigma)$ if $r$ has the column of length equal to $N_{c}$ in (A.9).

We make some comments on the parameter $n_{i \rightarrow j}(\sigma)$ in (5.2).

On the weak coupling side, the parameter $n_{i \rightarrow j}(\sigma)$ is interpreted as the number of open strings from the $i$-th brane to the $j$-th brane. On the strong coupling side, it is not clear whether we can introduce an open string as a probe, because non-maximal giants start oscillating by perturbing with infinitesimal energy; see figure 3. Moreover, the length of the probe string must be negligibly small, in order to maintain the gapless property of the dispersion relation. We will discuss a related issue in section 5.2.

The parameter $n_{i \rightarrow j}(\sigma)$ also counts the number of $Y$-fields, which should correspond to (part of) the angular momentum in $\mathrm{S}^{3}$ denoted by $J_{Y}=n_{Y}$. At strong coupling, the D-branes wrapping $S^{3}$ inside $S^{5}$ have the zero angular momentum in $\theta_{1}$ due to the static gauge (4.5). This conclusion is reasonable because we do not see $J_{Y} \sim O(1)$ at strong coupling, in agreement with the assumption $n_{Y} \ll n_{Z} \sim O\left(N_{c}\right)$ in the all-loop ansatz. If we still want to explain $J_{Y}$, we may also add a point particle rotating $\mathrm{S}^{3}$ carrying $J_{Y}$. This particle does not interact with D-branes at the leading order of large $N_{c} \cdot{ }^{14}$

When $p>2$ as in (5.1), we should find $p$ D3-branes oscillating individually, corresponding to the $\mathrm{U}(1)^{p}$ symmetry of the effective $\mathrm{U}(p)$ theory. The symmetry can be enhanced to non-abelian, e.g. $\mathrm{U}(2) \times \mathrm{U}(1)^{p-2}$, if some D-branes stay on top of each other at strong coupling, or if we give up the distant corners approximation at weak coupling. This point will be discussed in appendix E.

\footnotetext{
${ }^{14}$ If we think of the point particle as a closed string, this may also correspond to the term $n_{i i}(\sigma)$ in the effective $\mathrm{U}(p)$ theory Hamiltonian, which shows up in the subleading order of large $N_{c}[22,41]$.
} 


\subsection{On reflecting magnons}

At strong coupling, there is another known situation of open strings ending on a giant graviton, called the reflecting magnons in the literature [32]. An example can be depicted as

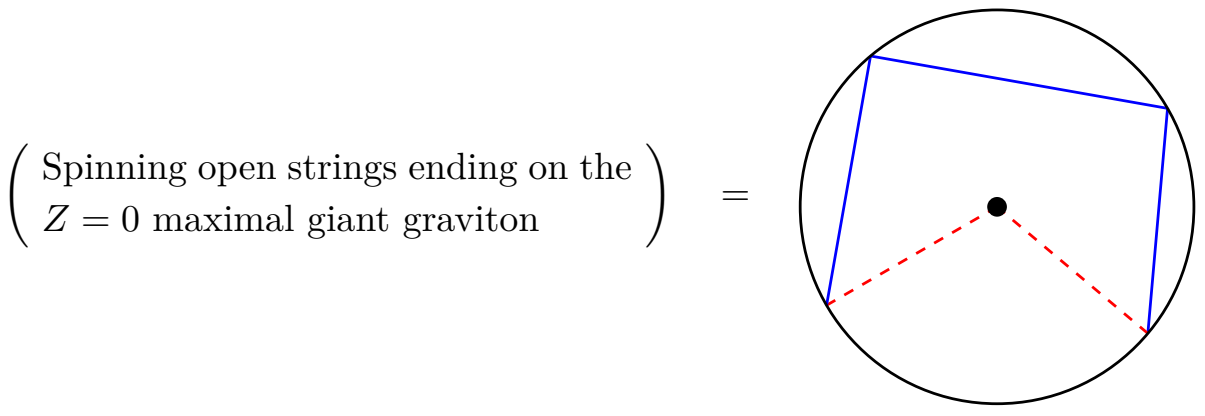

where the red dashed lines represent boundary magnons, and the blue thick lines represent bulk magnons. This figure is the same as the top view of figure 2 if the angular momentum $J_{\phi}$ takes the maximal value at $r=0$. The energy of open strings in (5.6) having a large angular momentum $J_{\text {string }}$ is given by the energy of an integrable open spin chain with $\mathfrak{s u}(2 \mid 2)$ symmetry [42] as

$$
E-J_{\text {string }}=\sum_{\alpha=L, R} \sqrt{Q_{\alpha}^{2}+\frac{\lambda}{\pi^{2}}}+\sum_{i=1}^{M} \sqrt{1+\frac{\lambda}{\pi^{2}} \sin ^{2} \frac{p_{i}}{2}}, \quad\left(N_{c} \gg J_{\text {string }} \gg 1\right)
$$

where $M$ is the number of bulk magnons. Note that the boundary terms can be interpreted as extra magnons with $p=\pi$.

At strong coupling $\lambda \gg 1$, the magnon energy (5.7) is equal to the sum of the open string length multiplied by the string tension. This dispersion relation cannot be gapless, because an open string should connect the equator and the north pole of $\mathrm{S}^{5}$.

At weak coupling, the system (5.6) is expected to be dual to a long operator attached to the determinant of $Z$ 's,

$$
\mathcal{O}_{\text {det }}=\sum_{\substack{i_{1}, i_{2}, \ldots, i_{N_{c}}, j_{1}, j_{2}, \ldots, j_{N_{c}}=1}}^{N_{c}} \epsilon_{j_{1} j_{2} \ldots j_{N_{c}}}^{i_{1} i_{2} \ldots i_{N_{c}}} Z_{i_{1}}^{j_{1}} Z_{i_{2}}^{j_{2}} \ldots Z_{i_{N_{c}-1}}^{j_{N_{c}-1}}\left(\chi_{L} \ldots Z Z \ldots \psi_{1} \ldots \psi_{2} \ldots Z Z \ldots \chi_{R}\right)_{i_{N_{c}}}^{j_{N_{c}}}
$$

where $\chi_{L}, \chi_{R}$ represent the boundary magnons and $\psi_{1}, \psi_{2}, \ldots$ represent the bulk magnon.

Consider the expansion of the determinant-like operator (5.8) in the Gauss graph basis. It is known that the determinant of $Z$ corresponds to $r=\llbracket N_{c} \rrbracket$, a single column of length $N_{c}$, and a single-trace operator is a linear combination of single hook Young diagrams [43]. ${ }^{15}$ Thus we expect that the determinant-like operator (5.8) should be expanded by $O^{R, r}(\sigma)$, where both $R$ and $r$ consist of a single hook attached to the column of length $O\left(N_{c}\right)$. We can generalize this system by introducing multiple giant gravitons. The Young diagrams

\footnotetext{
${ }^{15}$ This observation is true for the Schur polynomials, or the operators made out of $Z$ 's only. The singletrace operators with magnons may correspond to the restricted Schur polynomials whose $R$ is almost (but not exactly) a single-hook.
} 
$(R, r)$ for Gauss graph basis (A.9) should be modified as
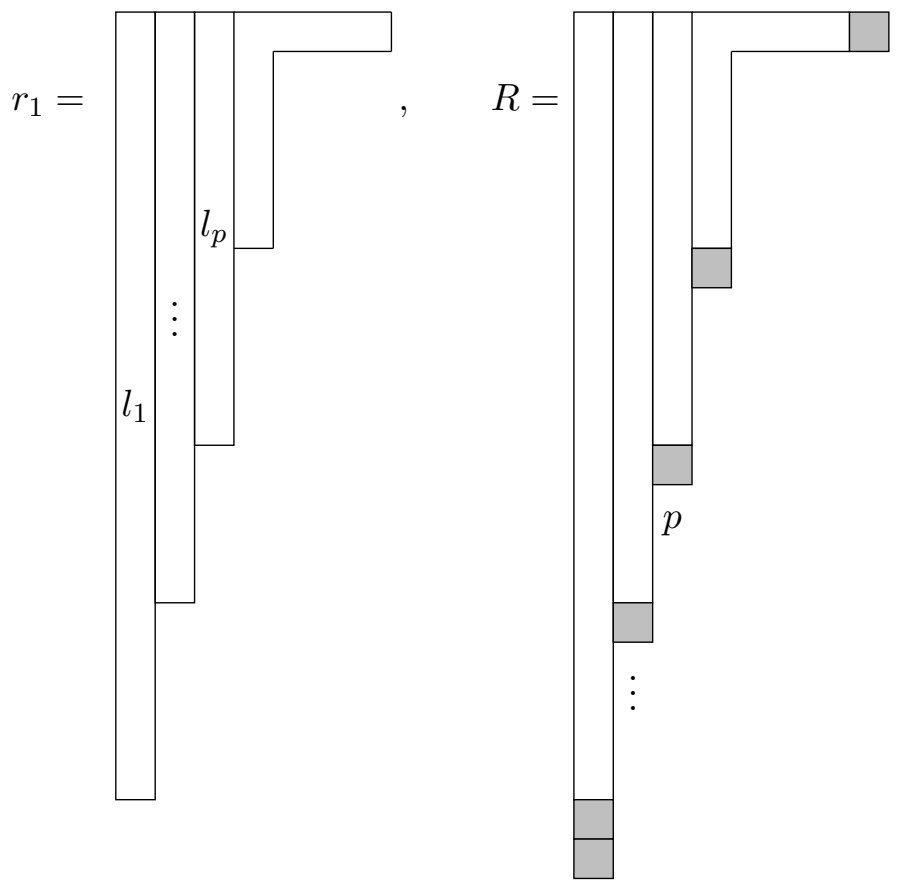

1

Recall that in the distant corners approximation, we can neglect the mixing of $Y$ fields in the different columns. Thus, the mixing matrix of the system (5.9) at large $N_{c}$ should factorize between the single-trace part and the effective $\mathrm{U}(p)$ theory part,

$$
\left.\left.\Delta-n_{Z}=\Delta \text { (Reflecting magnons }\right)+\Delta \text { (Oscillating giants }\right) .
$$

We can interpret the first term as (5.7) and the second term as (5.5) if $J_{\text {string }}$ (the length of a single-hook) is large.

Below are a few remarks on the $\mathfrak{s u}(2 \mid 2)$ symmetry.

We expect that the giant graviton possesses the residual superconformal symmetry $\mathfrak{p s u}(2 \mid 2)^{2}$, based on the $\kappa$-symmetric formulation of the D3-brane action on $\operatorname{AdS}_{5} \times \mathrm{S}^{5}$ [44]. However, we do not find any reasons that this symmetry should be promoted to the centrally-extended $\mathfrak{s u}(2 \mid 2)$. In other words, it is likely that the oscillating D-brane solutions are intrinsically non-BPS, and not centrally-extended BPS.

In [45], they constructed the $\mathfrak{s u}(2 \mid 2)$ generators of the effective $\mathrm{U}(p)$ theory. They proposed the central extension by

$$
\left\{\left(Q^{\alpha}{ }_{a}\right)_{i},\left(Q^{\beta}{ }_{b}\right)_{j}\right\}=\epsilon^{\alpha \beta} \epsilon_{a b} P_{i j} \quad\left\{\left(S^{a}{ }_{\alpha}\right)_{i},\left(S^{b}{ }_{\beta}\right)_{j}\right\}=\epsilon_{\alpha \beta} \epsilon^{a b} K_{i j}
$$

and interpreted $\Delta_{i j}^{ \pm}$as the centers

$$
P_{i j}=\alpha\left(d_{i}^{+}-d_{j}^{+}\right), \quad K_{i j}=\beta\left(d_{i}^{-}-d_{j}^{-}\right), \quad \Delta-J \stackrel{?}{=} \frac{1}{2} \sum_{i j} \sqrt{1+P_{i j} K_{i j}}
$$


which correspond to the second term of (5.10). However, the proposal (5.12) is inconsistent because 1.h.s. of (5.11) is symmetric under $(a, \alpha, i) \leftrightarrow(b, \beta, j)$ whereas the r.h.s. is antisymmetric. $^{16}$

An alternative approach to the question of the central extension is as follows. Here we gave several expressions of the D-brane energy; the conjectured all-loop formula (5.1), $\Delta$ (Oscillating giants) in (5.10), and the boundary term in the spin chain energy (5.7). Only the last expression takes the square-root form inherited from the centrally extended $\mathfrak{s u}(2 \mid 2)$ symmetry. ${ }^{17}$ For the other cases we do not find square-roots, and one possible reason is the wrapping corrections to the $Z=0$ brane [46]. Roughly speaking, the oscillating giants without open strings would correspond to the $J_{\text {string }} \rightarrow 0$ limit of the integrable spin chain. In other words, it may be possible to refine the proposal (5.12) by studying the states like (5.9) in the limit of $J_{\text {string }} \gg 1 .^{18}$

\section{Summary}

In this paper, we studied a non-planar large $N_{c}$ limit of $\mathcal{N}=4 \mathrm{SYM}$ as a new example of the AdS/CFT correspondence. First, we reviewed the Hamiltonian of an effective $\mathrm{U}(p)$ theory coming from the perturbative dilatation operator acting on the Gauss graph basis. When $p=2$, this model is related to the finite harmonic oscillator. Second, we proposed an all-loop ansatz based on the effective $\mathrm{U}(p)$ theory. We found mutually commuting charges generated by the difference operators. By taking the continuum limit, we argue that higher loop terms should be proportional to the one-loop result.

In our all-loop ansatz, the harmonic oscillators remain non-vanishing in the large $N_{c}$ limit, giving a gapless dispersion relation. In particular, it indicates that non-BPS excited giant gravitons should be continuously connected to the BPS giant graviton at strong coupling.

We investigated the classical D3-brane action on $\mathrm{AdS}_{5} \times \mathrm{S}^{5}$ and found that a nonmaximal spherical giant graviton can be excited in a gapless way. We argued that this new oscillating brane with KK modes on $\mathrm{S}^{3}$ is a good candidate for the AdS/CFT dictionary which corresponds to the harmonic oscillator in the effective $\mathrm{U}(p)$ theory.

Possible future directions are sketched as follows.

One direction is to investigate this correspondence further. At weak coupling, the mixing matrix on the Gauss graph basis should be evaluated in a more general setup. This includes higher loop effects, a larger set of operators including the $\mathfrak{s l}(2)$ sector [47], and the corrections from higher orders in $n_{Y} / n_{Z}$ [48]. At strong coupling, the dynamics of D3-brane on $\mathrm{AdS}_{5} \times \mathrm{S}^{5}$ should be studied in a comprehensive way. This includes to resum the $\epsilon$ series in the $k=0$ solution (4.27), and to investigate the non-abelian DBI action [49]. The non-abelian analysis of the pp-wave background [29], the F1-D3 system [50] and the D1-D3 system [51] may be helpful. A closely related method is the matrix regularization of the worldvolume theory in the pp-wave background [52-54], which should capture part of the energy spectrum at strong coupling.

\footnotetext{
${ }^{16}$ In addition, (5.12) lacks $n_{i j}(\sigma)$, and disagrees with the two-loop result if $\alpha \beta=O(1)$.

${ }^{17}$ The bulk and boundary terms are essentially the same in the collective coordinate approach [33-35].

${ }^{18}$ We thank an anonymous referee of JHEP for this comment.
} 
It is interesting to generalize the computation of the three-point function between two giants and one graviton, into the correlators with oscillating giants [55-57], which serves as a non-trivial check of our proposal.

Another direction is to determine the general spectrum of the Hamiltonian of the effective $\mathrm{U}(p)$ theory. This includes solving the finite oscillator for $p>2$, finding consistent wave-functions for AdS giants, and understanding the structure of $1 / N_{c}$ corrections.

The role of the superconformal symmetry needs to be examined. In particular, the $\mathcal{N}=4$ SYM theory can be deformed while keeping $\mathfrak{s u}(2 \mid 2)^{2}[58]$. It is worth investigating the corresponding deformation at strong coupling and finding the relation to the system of giant gravitons in $\mathrm{AdS}_{5} \times \mathrm{S}^{5}$.

A challenging question is whether the "non-planar integrability" can be found at strong coupling. One starting point is the $\kappa$-symmetric D3-brane action in $\mathrm{AdS}_{5} \times \mathrm{S}^{5}$ [44]. Then, through the reduction to two-dimensions (4.7), we may be able to find a classical integrable system. The two-dimensional reduction may not be necessary if one can construct an integrable system in $4 \mathrm{~d}$ along the line of [59-61].

\section{Acknowledgments}

RS thanks the organizers of the workshops YITP-W-20-03 on Strings and Fields 2020, and Online 2020 NTU-Kyoto high energy physics workshop for stimulating discussions. He is grateful to Robert de Mello Koch, Arkady Tseytlin and Keisuke Okamura for comments on the manuscript. This research is supported by NSFC grant no. 12050410255 .

\section{A Review of the Gauss graph basis}

We briefly review the construction of the Gauss graph basis, and how it simplifies the action of the perturbative one-loop dilatation operators of $\mathcal{N}=4 \mathrm{SYM}$.

\section{A.1 Notation}

Let $S_{L}$ be a permutation group of degree $L$. Its irreducible representations are labeled by a partition (Young diagram) $R \vdash L$, whose dimensions are denoted by $d_{R} . D_{I J}^{R}(\sigma)$ denotes the matrix representation of $\sigma$ in the irreducible representation $R$ with the component $(I, J)$, where $I, J=1,2, \ldots, d_{R}$.

Consider the restriction $S_{L} \downarrow\left(S_{m} \otimes S_{n}\right)$ with $m+n=L$. We denote the irreducible decomposition by

$$
R=\bigoplus_{\substack{r \vdash m \\ s \vdash n}} \bigoplus_{\nu=1}^{g(r, s ; R)}(r \otimes s)_{\nu}
$$

where $\nu$ is a multiplicity label and $g(r, s ; R)$ is the Littlewood-Richardson coefficient. The branching coefficients are defined by the overlap between the components

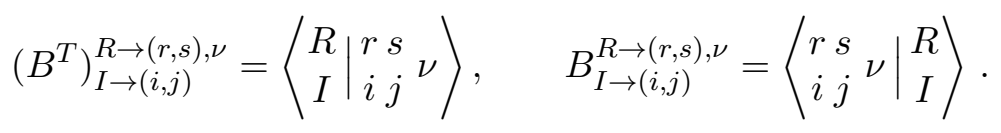

See [9] for the properties of these quantities. 
We denote partitions of an integer, or Young diagrams, in two ways. The symbol $y=\llbracket l_{1}, l_{2}, \ldots, l_{p} \rrbracket$ means that the $i$-th column of the Young diagram $y$ has the length $l_{i}$. The symbol $y=\left[m_{1}, m_{2}, \ldots, m_{q}\right]$ means that the $j$-th row of $y$ has the length $m_{j}$. It follows that

$$
y \vdash L=\sum_{i=1}^{p} l_{i}=\sum_{j=1}^{q} m_{j}, \quad l_{1} \geq l_{2} \geq \cdots \geq l_{p}, \quad m_{1} \geq m_{2} \geq \cdots \geq m_{q} .
$$

\section{A.2 Distant corners approximation}

We introduce the collective index

$$
\left(Z^{\otimes n}\right)_{\vec{\jmath}}^{\vec{\imath}}=Z_{j_{1}}^{i_{1}} Z_{j_{2}}^{i_{2}} \ldots Z_{j_{n}}^{i_{n}}, \quad\left(U_{\alpha}\right)_{\vec{k}}^{\vec{\jmath}}=\delta_{k_{\alpha(1)}}^{j_{1}} \delta_{k_{\alpha(2)}}^{j_{2}} \ldots \delta_{k_{\alpha(n)}}^{j_{n}} \quad\left(\alpha \in S_{n}\right)
$$

with $i_{p}, j_{p}=1,2, \ldots, N_{c}$. The matrix $U_{\alpha}$ satisfies the composition rules

$$
\left(U_{\alpha}\right)_{\vec{\jmath}}^{\vec{\imath}}\left(U_{\beta}\right)_{\vec{\imath}}^{\vec{k}}=\left(U_{\alpha \beta}\right)_{\vec{\jmath}}^{\vec{k}}, \quad\left(U_{\alpha}\right)_{\vec{\jmath}}^{\vec{\imath}}\left(U_{\beta}\right)_{\vec{k}}^{\vec{\jmath}}=\left(U_{\beta \alpha}\right)_{\vec{k}}^{\vec{\imath}} \operatorname{tr}_{n}\left(U_{\alpha}\right)=N_{c}^{C(\alpha)}
$$

We denote multi-trace operators in the $\mathfrak{s u}(2)$ sector by

$$
\operatorname{tr}_{L}\left(U_{\alpha} \cdot Y^{\otimes n_{Y}} Z^{\otimes n_{Z}}\right)=\sum_{i_{1}, i_{2}, \ldots, i_{L}=1}^{N_{c}} Y_{i_{\alpha(1)}}^{i_{1}} Y_{i_{\alpha(2)}}^{i_{2}} \ldots Y_{i_{\alpha\left(n_{Y}\right)}}^{i_{n_{Y}}} Z_{i_{\alpha\left(n_{Y}+1\right)}}^{i_{n_{Y}+1}} Z_{i_{\alpha\left(n_{Y}+2\right)}}^{i_{n_{Y}+2}} \ldots Z_{i_{\alpha(L)}}^{i_{L}}
$$

with $L=n_{Y}+n_{Z}$ and $\alpha \in S_{L}$. We define the restricted Schur basis of operators by

$$
\begin{aligned}
\mathcal{O}^{R,(r, s), \nu_{+}, \nu_{-}} & =\frac{1}{n_{Y} ! n_{Z} !} \sum_{\alpha \in S_{L}} \chi^{R,(r, s), \nu_{+}, \nu_{-}}(\alpha) \operatorname{tr}_{L}\left(U_{\alpha} \cdot Y^{\otimes n_{Y}} Z^{\otimes n_{Z}}\right) \\
\chi^{R,(r, s), \nu_{+}, \nu_{-}}(\alpha) & =\sum_{I, J=1}^{d_{R}} \sum_{i=1}^{d_{r}} \sum_{j=1}^{d_{s}} B_{I \rightarrow(i, j)}^{R \rightarrow(r, s) \nu_{+}}\left(B^{T}\right)_{J \rightarrow(i, j)}^{R \rightarrow(r, s), \nu_{-}} D_{I J}^{R}(\alpha)
\end{aligned}
$$

coming from the restriction $S_{L} \downarrow\left(S_{n_{Y}} \otimes S_{n_{Z}}\right)$.

It is expected that the half-BPS operators dual to $p$ spherical giant gravitons consist of $p$ long columns, with $n_{Z}=O\left(N_{c}\right)$ with $N_{c} \gg 1$. Non-BPS operators can be constructed by attaching $Y$ fields. We write $r=\llbracket l_{1}, l_{2}, \ldots, l_{p} \rrbracket$ where $l_{i}$ is the length of the $i$-th column. In the distant corners approximation, we assume that $l_{i}-l_{i-1} \gg 1$, so that the corners of 
$r$ are well separated. Therefore, we typically work with Young diagrams
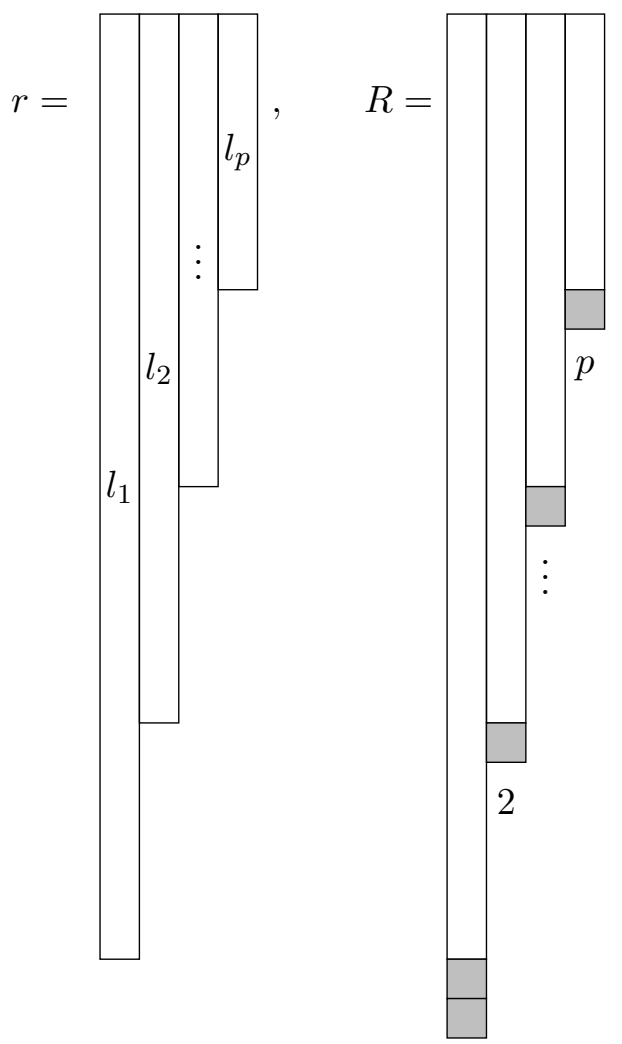

1

where we construct $R$ by adding $s \vdash n_{Y}$ (gray boxes) to $r \vdash n_{Z}$ (white boxes).

\section{A.3 Gauss graph basis}

We introduce the Gauss graph basis following [16].

\section{A.3.1 Skew Young diagrams}

We can specify the representation of $Y$ fields in two ways, $s$ or $R / r$. The states of $s$ are labeled by the standard Young tableaux, and those of $R / r$ are by the skew Young tableaux. In the restricted Schur polynomial (A.8), we may keep track of which box of $s$ goes to which box of $R / r$, before summing over the indices $(I, J, i, j)$.

In the distant corners approximation, $R / r$ consists of $p$ columns well separated from each other. This indicates that only the column position, $1,2, \ldots, p$, should be important in finding the eigenstates of the perturbative dilatation operator of $\mathcal{N}=4 \mathrm{SYM}$. 
Consider an example of $p=3$, with $s=\llbracket 4,2,1 \rrbracket$ and $R / r=\llbracket 3,2,2 \rrbracket$. We parameterize a state of $s$ and $R / r$ using only the column labels, as

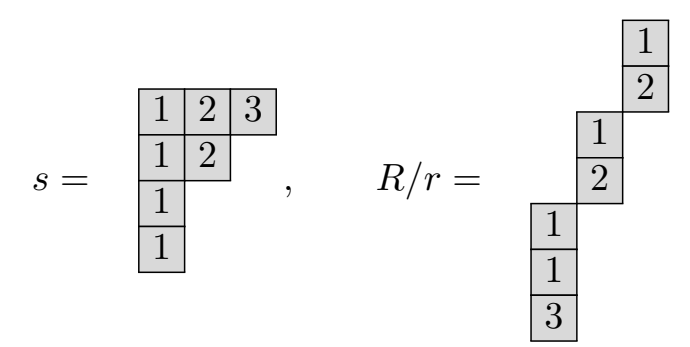

In other words, we project the standard Young diagrams of shape $s \vdash n_{Y}$ onto the trivial (totally symmetric) representation of

$$
H=S_{s_{1}} \otimes S_{s_{2}} \otimes \ldots S_{s_{p}} \subset S_{n_{Y}}, \quad s=\llbracket s_{1}, s_{2}, \ldots, s_{p} \rrbracket .
$$

The group $H$ is an extra symmetry that emerges in the distant corners approximation [23]. We should refine the label of the restricted Schur operator $\mathcal{O}^{R,(r, s), \nu_{+}, \nu_{-}}$by adding $\vec{s}=\left(s_{1}, s_{2}, \ldots, s_{p}\right)$ which specifies how $s \vdash n_{Y}$ shows up in the skew Young diagram $R / r$.

\section{A.3.2 Adjacency matrix}

We can define the adjacency matrix $n_{i \rightarrow j}$ by counting how many $i$ 's appear in the $j$-th column of the skew tableau $R / r$. In the above example (A.10) we find

$$
\left\{n_{i \rightarrow j}\right\}=\left(\begin{array}{lll}
2 & 1 & 1 \\
0 & 1 & 1 \\
1 & 0 & 0
\end{array}\right) .
$$

The adjacency matrix satisfies a conservation law. When a box with the label $i$ goes to the $j$-th column $(j \neq i)$, then there must be a box $k$ which comes to the $i$-th column. This implies the relation called the Gauss law constraints,

$$
\sum_{j=1}^{p} n_{i \rightarrow j}=\sum_{j=1}^{p} n_{j \rightarrow i} .
$$

Intuitively, we may regard the diagonal elements $n_{i \rightarrow i}$ as the number of closed strings on the $i$-th brane, and the off-diagonal elements $n_{i \rightarrow j}$ as the number of open strings between the $i$-th and $j$-th brane.

Here we defined the adjacency matrix from a skew Young diagram. The skew Young diagram is in one-to-one correspondence with the Gelfand-Testlin basis, as discussed in [15].

\section{A.3.3 Permutation and the double coset}

We can determine the adjacency matrix $\left\{n_{i \rightarrow j}\right\}$ from a permutation element $\sigma \in S_{n_{Y}}$ as follows. Given $s=\llbracket s_{1}, s_{2}, \ldots, s_{p} \rrbracket$, we introduce a state

$$
\begin{aligned}
|\vec{s}\rangle & \equiv|1 \bar{\otimes} \ldots \bar{\otimes} 1\rangle \otimes|2 \bar{\otimes} \ldots \bar{\otimes} 2\rangle \otimes \cdots \otimes|p \bar{\otimes} \ldots \bar{\otimes} p\rangle \\
& =\left|1^{\bar{\otimes} s_{1}} \otimes 2^{\bar{\otimes} s_{2}} \otimes \cdots \otimes p^{\bar{\otimes} s_{p}}\right\rangle
\end{aligned}
$$


where $\bar{\otimes}$ represents the symmetrized tensor product and $\otimes$ is the usual tensor product. The symmetrization is equivalent to a sum over the states in $V_{p}^{\otimes n_{Y}}$ where $V_{p}=\{1,2, \ldots, p\}$. We can permute the state $|\vec{s}\rangle$ by applying $\sigma \in S_{n_{Y}}$ to each summand as

$$
\begin{aligned}
|\sigma, \vec{s}\rangle= & \left|\sigma(1) \otimes \sigma(2) \otimes \cdots \otimes \sigma\left(n_{Y}\right)\right\rangle_{\mathrm{symm}} \\
\equiv & \left|\sigma(1) \bar{\otimes} \ldots \bar{\otimes} \sigma\left(s_{1}\right)\right\rangle \otimes\left|\sigma\left(s_{1}+1\right) \bar{\otimes} \ldots \bar{\otimes} \sigma\left(s_{1}+s_{2}\right)\right\rangle \otimes \ldots \\
& \otimes\left|\sigma\left(n_{Y}-s_{p}+1\right) \bar{\otimes} \ldots \bar{\otimes} \sigma\left(s_{n_{Y}}\right)\right\rangle .
\end{aligned}
$$

This result consists of $p$ tensor product of symmetrized components. It makes sense to count the number of $i$ 's in the $j$-th symmetrized component, and call it $n_{i \rightarrow j}$.

Owing to the symmetrization, the action of the permutation $\sigma \in S_{n_{Y}}$ reduces to the action of an element in the double coset $\bar{\sigma} \in H \backslash S_{n_{Y}} / H$,

$$
\bar{\sigma}=\frac{1}{|H|} \sum_{\gamma_{1}, \gamma_{2} \in H} \gamma_{1} \sigma \gamma_{2}, \quad \sigma \in S_{n_{Y}} .
$$

We can compute $n_{i \rightarrow j}$ graphically as

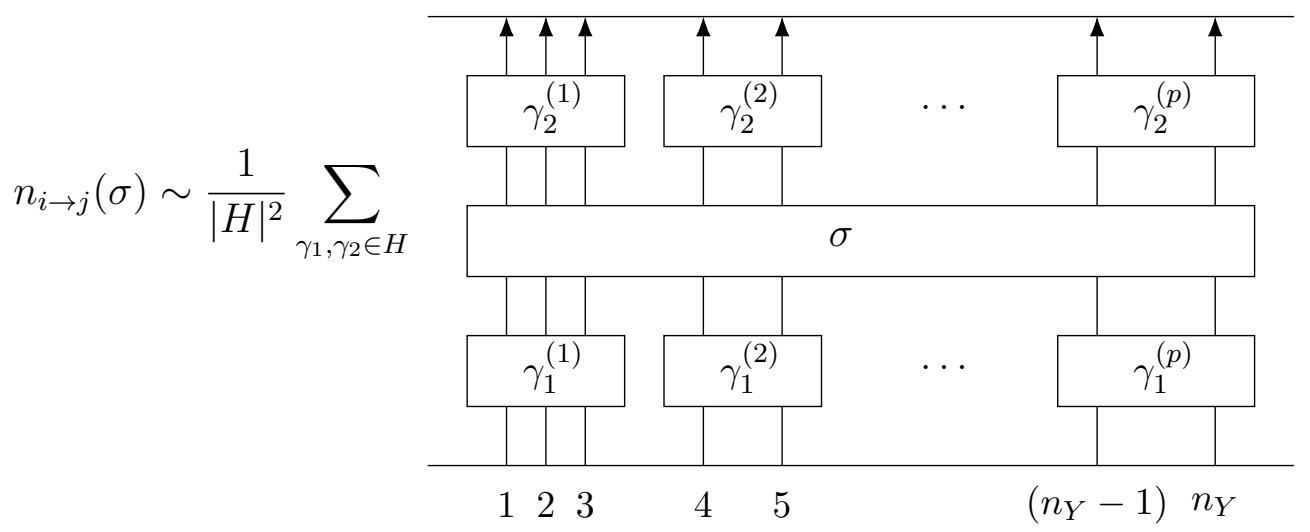

which corresponds to $\left(s_{1}, s_{2}, \ldots, s_{p}\right)=(3,2, \ldots, 2)$.

\section{A.3.4 Operator mixing in the Gauss graph basis}

We define operators in the Gauss graph basis by ${ }^{19}$

$$
O^{R, r}(\sigma)=|H| \sqrt{n_{Y} !} \sum_{j, k} \sum_{s \vdash n_{Y}} \sum_{\nu_{-}, \nu_{+}} D_{j k}^{s}(\sigma) B_{j}^{s \rightarrow 1_{H}, \nu_{-}}\left(B^{T}\right)_{k}^{s \rightarrow 1_{H}, \nu_{+}} \mathcal{O}^{R,(r, s), \nu_{+}, \nu_{-}} .
$$

We consider the case $n_{Z}=O\left(N_{c}\right)$ and $n_{Y}=O(1)$, which should correspond to excited multiple giant gravitons. The one-loop dilatation acting on the restricted Schur polynomial factorizes into the mixing of $Y$ 's and the mixing of $Z$ 's at the leading order of large $N_{c}{ }^{20}$ As shown in [16], the mixing of $Y$ 's can be solved by taking the Gauss graph basis. The eigenvalues are labeled by the symmetrized adjacency matrix,

$$
n_{i j}(\sigma) \equiv n_{i \rightarrow j}(\sigma)+n_{j \rightarrow i}(\sigma), \quad n_{i j}(\sigma)=n_{j i}(\sigma) .
$$

\footnotetext{
${ }^{19}$ Our definition looks slightly different from [16] because our restricted Schur basis (A.7) is not normalized.

${ }^{20}$ The factorization property is violated at the subleading order of $n_{Y} / n_{Z}$ [48].
} 
The mixing of $Z$ 's changes the shape of $r=\llbracket l_{1}, l_{2}, \ldots, l_{p} \rrbracket$. We use the simplified notation

$$
O(\vec{l})=O\left(l_{1}, l_{2}, \ldots, l_{p}\right), \quad \sum_{i=1}^{p} l_{i}=n_{Z}
$$

in place of (A.18).

\section{B Explicit one-loop spectrum}

We studying the spectrum of $D_{1}^{G}=-\mathcal{H}_{1}$ following $[14,15]$ with minor improvement.

We consider both continuum and discrete cases. The dilatation operator reduces to a set of harmonic oscillators with boundary conditions in the continuum limit (2.29). In order to determine the dilatation spectrum before taking the limit, we solve the discrete case. The discrete case has been solved for $p=2$ by using the finite oscillator [62,63]. The spectra of the two cases agree, implying that the one-loop dimensions do not depend on the details of the continuum limit.

\section{B.1 Continuum case}

The spectrum of one-loop dilatation in the continuum limit for general $p$ has been studied in [15]. In this limit, we find $D_{1}^{G}=-\mathcal{H}_{1} \rightarrow-\mathcal{D}$ where

$$
\mathcal{D} F(\vec{y})=E\left(\left\{n_{i j}\right\}\right) F(\vec{y}), \quad \mathcal{D} \equiv \sum_{i \neq j}^{p} n_{i j}(\sigma) \mathcal{D}_{i j} .
$$

We solve this equation in the region of $\left\{y_{i}\right\}$ given in (2.30). We rewrite the differential operator $\mathcal{D}_{i j}$ in $(2.33)$ as

$$
\mathcal{D}_{i j} F=\left(A^{+}\left(y_{i j}\right) A^{-}\left(y_{i j}\right)+\frac{1}{2}\right) F, \quad A^{ \pm}(y)=\frac{1}{\sqrt{\alpha}}\left\{\frac{\alpha y}{2} \pm \frac{\partial}{\partial y}\right\}
$$

where $y_{i j}=y_{i}-y_{j}$. The new differential operators $\left\{A^{ \pm}(y)\right\}$ satisfy

$$
\left[A^{+}\left(y_{i j}\right), A^{-}\left(y_{k l}\right)\right]=\delta_{i k}+\delta_{j l}-\delta_{i l}-\delta_{j k} .
$$

The operator $\mathcal{D}_{i j}$ has the symmetry

$$
\left[\mathcal{D}_{i j}, \Lambda^{ \pm}\right]=0, \quad \Lambda^{+} \equiv \sum_{i=1}^{p} y_{i}, \quad \Lambda^{-} \equiv \sum_{i=1}^{p} \frac{\partial}{\partial y_{j}} .
$$

Thus, $\Lambda^{ \pm}$represent the zero modes of $\mathcal{D}$. Roughly speaking, $\Lambda^{ \pm}$correspond to the addition or removal of a box from each of the $p$ columns, suggesting that the spectrum of $\mathcal{H}_{1}$ depends only on the difference of column lengths.

From (B.2) one finds that the eigenvalues of $\mathcal{D}_{i j}$ are written as $\left(m+\frac{1}{2}\right)$. The mode number $m$ should be chosen so that the variables $y_{i j}$ satisfy the Young diagram constraints (2.30). These constraints are expressed in terms of the variables $\left(y_{12}, \ldots, y_{p-1, p}, y_{p}\right)$ as

$$
y_{12} \geq 0, \quad y_{23} \geq 0, \quad \ldots, \quad y_{p-1, p} \geq 0, \quad y_{p} \geq 0 .
$$


Consider the operator ${ }^{21}$

$$
\tilde{\mathcal{D}} \equiv \mathcal{D}-n_{Y}=2 \sum_{i<j}^{p} n_{i j}(\sigma) A^{+}\left(y_{i j}\right) A^{-}\left(y_{i j}\right), \quad A^{ \pm}\left(y_{i j}\right)=\sum_{k=i}^{j-1} A^{ \pm}\left(y_{k, k+1}\right) .
$$

This operator can be written as the following quadratic form

$$
\tilde{\mathcal{D}}=2 \sum_{u, v=1}^{p-1} \mathscr{M}_{u, v} A^{+}\left(y_{u, u+1}\right) A^{-}\left(y_{v, v+1}\right)
$$

where

$$
\mathscr{M}_{u, v}= \begin{cases}\sum_{k=1}^{v} \sum_{l=u+1}^{p} n_{k, l} & (u \geq v) \\ \sum_{k=1}^{u} \sum_{l=v+1}^{p} n_{k, l} & (u<v) .\end{cases}
$$

Note that $\mathscr{M}$ is a $(p-1) \times(p-1)$ matrix which depends on $\left\{n_{i, j}\right\}$ with $i<j$. By diagonalizing $\mathscr{M}$, we obtain the non-zero eigenvalues of $\tilde{\mathcal{D}}$.

$$
\tilde{\lambda}_{u} \delta_{u v}=\left(\mathscr{S} \mathscr{M} \mathscr{S}^{T}\right)_{u v}, \quad \tilde{\mathcal{D}}=2 \sum_{a=1}^{p-1} \tilde{\lambda}_{a} A^{+}\left(\tilde{z}_{a}\right) A^{-}\left(\tilde{z}_{a}\right) .
$$

The new coordinates $\left\{\tilde{z}_{a}\right\}$ are written as

$$
y_{a, a+1}=\sum_{b=1}^{p}\left(\mathscr{S}^{T}\right)_{a b} \tilde{z}_{a} .
$$

We define the vacuum of $\mathcal{H}_{1}$ by requiring

$$
A^{-}\left(\tilde{z}_{a}\right)|0\rangle=0 \quad(\forall a)
$$

and define excited states by applying the creation operators $A^{+}\left(\tilde{z}_{a}\right)$. We solve the Young diagram constraints (B.5) as follows. The boundary of the constraints lies on $y_{a, a+1}=0$, or equivalently $\tilde{z}_{a}=0$. The wave function $\psi\left(\left\{\tilde{z}_{a}\right\}\right)$ should vanish at $\tilde{z}_{a}=0$ for all $a$, because it is ill-defined in the region $y_{a, a+1}<0$. Recall that the creation operators are parity odd,

$$
A^{+}\left(-\tilde{z}_{a}\right)=-A^{+}\left(\tilde{z}_{a}\right) .
$$

The physical states should contain an odd number of creation operators for each $a$. Thus, the spectrum of $\tilde{\mathcal{D}}$ is

$$
\tilde{\mathcal{D}} \psi_{\vec{m}}\left(\left\{\tilde{z}_{a}\right\}\right)=2 \sum_{a=1}^{p-1}\left(2 m_{a}+1\right) \tilde{\lambda}_{a} \psi_{\vec{m}}\left(\left\{\tilde{z}_{a}\right\}\right), \quad m_{a} \in \mathbb{Z}_{\geq 0}
$$

The eigenvalues of the original equation (B.1) are

$$
E\left(\left\{n_{i j}\right\}\right)=n_{Y}+2 \sum_{a=1}^{p-1}\left(2 m_{a}+1\right) \tilde{\lambda}_{a}\left(\left\{n_{i j}\right\}\right) .
$$

\footnotetext{
${ }^{21}$ We used $\sum_{i \neq j} n_{i j}(\sigma)=2 n_{Y}$.
} 


\section{B.2 Discrete case}

The main difficulty in computing the discrete spectrum lies in how to impose the Young diagram constraints. The Fock space created by the oscillator representation of $\mathcal{H}_{1}$ in (2.17) is not useful, because it does not immediately solve the constraints. Instead, we directly look for the wave functions. Some functional identities are summarized in appendix C.

For simplicity, we consider the case of $p=2$. We take the linear combination of $O\left(l_{1}, l_{2}\right)$ in $(2.9)$ as

$$
\mathcal{O}_{f}=\sum_{x=-l_{2}}^{\left\lceil\left(l_{1}-l_{2}\right) / 2\right\rceil} f(x) O\left(l_{1}-x, l_{2}+x\right) .
$$

The operator $\mathcal{H}_{1,12}$ in $(2.17)$ acts on $\mathcal{O}_{f}$ as

$$
\begin{aligned}
\mathcal{H}_{1,12} \mathcal{O}_{f}= & \left(h\left(1, l_{1}\right)+h\left(2, l_{2}\right)\right) \mathcal{O}_{f}-\sqrt{h\left(1, l_{1}\right) h\left(2, l_{2}+1\right)} \sum_{x} f(x) O\left(l_{1}-x-1, l_{2}+x+1\right) \\
& -\sqrt{h\left(1, l_{1}+1\right) h\left(2, l_{2}\right)} \sum_{x} f(x) O\left(l_{1}-x+1, l_{2}+x-1\right)
\end{aligned}
$$

which gives the following discrete eigenvalue equation

$$
\begin{aligned}
\mathcal{H}_{1,12} & =h\left(1, l_{1}\right)+h\left(2, l_{2}\right)-\sqrt{h\left(1, l_{1}\right) h\left(2, l_{2}+1\right)} e^{-\partial_{x}}-\sqrt{h\left(1, l_{1}+1\right) h\left(2, l_{2}\right)} e^{+\partial_{x}} \\
\mathcal{H}_{1,12} f(x) & =E f(x) .
\end{aligned}
$$

This equation determines the eigenvalue of $D_{1}^{G}=-\mathcal{H}_{1}$ at $p=2$ as

$$
D_{1}^{G}=-2 n_{12} E
$$

where we used $n_{12}=n_{21}$.

We solve (B.17) by relating it to a finite oscillator [62,63]. Let us take a basis of states in the irreducible representations of $\mathfrak{s u}(2)$,

$$
J_{3}\left|j, j_{3}\right\rangle=j_{3}\left|j, j_{3}\right\rangle, \quad\left(j_{3}=-j,-j+1, \ldots, j\right)
$$

and a rotated basis

$$
J_{1}\left|j, j_{1}\right\rangle_{1}=j_{1}\left|j, j_{1}\right\rangle_{1}, \quad\left(j_{1}=-j,-j+1, \ldots, j\right) .
$$

In view of the effective $\mathrm{U}(p)$ theory, the $\mathfrak{s u}(2)$ generators can be interpreted as

$$
J_{1}=\frac{1}{2}\left(d_{1}^{+} d_{2}^{-}+d_{2}^{+} d_{1}^{-}\right), \quad J_{2}=\frac{i}{2}\left(d_{1}^{+} d_{2}^{-}-d_{2}^{+} d_{1}^{-}\right), \quad J_{3}=\frac{1}{2}\left(d_{1}^{+} d_{1}^{-}-d_{2}^{+} d_{2}^{-}\right) .
$$

The generator $\left(2 J_{1}\right)$ is identical to $\mathcal{J}_{12}$ in $(3.7)$. The rotation matrix is given by

$$
{ }_{1}\left\langle j, j_{1} \mid j, j_{3}\right\rangle=\frac{(-1)^{j+j_{3}}}{2^{j}} \sqrt{\left(\begin{array}{c}
2 j \\
j+j_{3}
\end{array}\right)\left(\begin{array}{c}
2 j \\
j+j_{1}
\end{array}\right)}{ }_{2} F_{1}\left(-j-j_{3},-j-j_{1} ;-2 j ; 2\right) .
$$

The $\mathfrak{s u}(2)$ generators acts on the states $\left|j, j_{1}\right\rangle_{1}$ in the standard way,

$$
J_{1}\left|j, j_{1}\right\rangle_{1}=j_{1}\left|j, j_{1}\right\rangle_{1}, \quad J_{ \pm}\left|j, j_{1}\right\rangle_{1}=\sqrt{\left(j \mp j_{1}\right)\left(j \pm j_{1}+1\right)}\left|j, j_{1} \pm 1\right\rangle_{1} .
$$


We define

$$
n_{Z} \equiv l_{1}+l_{2}, \quad l_{12}=l_{1}-l_{2}
$$

and assume $n_{Z} \leq 2 N_{c}$, which is trivial at $p=2$. Let us assign

$$
j=N_{c}-\frac{n_{Z}-1}{2}, \quad j_{1}=\frac{-l_{12}-1}{2}, \quad j+j_{3}=m-1
$$

where the new variables run the ranges

$$
n_{Z}-2 N_{c}-2 \leq l_{12} \leq 2 N_{c}-n_{Z}, \quad 1 \leq m \leq 2 N_{c}-n_{Z}+2 .
$$

Note that

$$
l_{12}=n_{Z}-2 N_{c}-2 \quad \Leftrightarrow \quad l_{2}=N_{c}+1, \quad l_{12}=2 N_{c}-n_{Z} \quad \Leftrightarrow \quad l_{1}=N_{c} .
$$

The equations (B.23) become

$$
\begin{aligned}
& J_{1}\left|N_{c}-\frac{n_{Z}-1}{2}, \frac{-l_{12}-1}{2}\right\rangle_{1}=\frac{-l_{12}-1}{2}\left|N_{c}-\frac{n_{Z}-1}{2}, \frac{-l_{12}-1}{2}\right\rangle_{1} \\
& J_{+}\left|N_{c}-\frac{n_{Z}-1}{2}, \frac{-l_{12}-1}{2}\right\rangle_{1}=\sqrt{\left(N_{c}-l_{2}+1\right)\left(N_{c}-l_{1}+1\right)}\left|N_{c}-\frac{n_{Z}-1}{2}, \frac{-l_{12}+1}{2}\right\rangle_{1} \\
& J_{-}\left|N_{c}-\frac{n_{Z}-1}{2}, \frac{-l_{12}-1}{2}\right\rangle_{1}=\sqrt{\left(N_{c}-l_{1}\right)\left(N_{c}-l_{2}+2\right)}\left|N_{c}-\frac{n_{Z}-1}{2}, \frac{-l_{12}-3}{2}\right\rangle_{1} .
\end{aligned}
$$

The last two lines agree with the off-diagonal terms in (B.17) with the help of (2.10).

We can immediately solve (B.17) by relating the wave function to the rotation matrix (B.22). If we define

$$
\begin{aligned}
F_{m}\left(l_{1}, l_{2}\right)= & (-1)^{m-1} 2^{-N_{c}+\frac{1}{2}\left(n_{Z}-1\right)} \sqrt{\left(\begin{array}{c}
2 N_{c}-n_{Z}+1 \\
m-1
\end{array}\right)\left(\begin{array}{c}
2 N_{c}-n_{Z}+1 \\
N_{c}-l_{1}
\end{array}\right)} \times \\
& { }_{2} F_{1}\left(-m+1,-N_{c}+l_{1} ;-2 N_{c}+n_{Z}-1 ; 2\right) .
\end{aligned}
$$

this function satisfies the recursion relation

$$
\begin{aligned}
& \sqrt{h\left(1, l_{1}\right) h\left(2, l_{2}+1\right)} F_{m}\left(l_{1}-1, l_{2}+1\right)+\sqrt{h\left(1, l_{1}+1\right) h\left(2, l_{2}\right)} F_{m}\left(l_{1}+1, l_{2}-1\right) \\
& -\left(h\left(1, l_{1}\right)+h\left(2, l_{2}\right)\right) F_{m}\left(l_{1}, l_{2}\right)=-2 m F_{m}\left(l_{1}, l_{2}\right)
\end{aligned}
$$

which is equivalent to the discrete eigenvalue equation (B.17) with $E=2 m$.

We should impose the Young diagram constraints at $p=2$, namely

$$
\begin{cases}N_{c} \geq l_{1} \geq l_{2} \geq\left(N_{c}-l_{1}\right) & \left(n_{Z} \geq N_{c}\right) \\ l_{1} \geq l_{2} \geq 0 & \left(n_{Z} \leq N_{c}\right) .\end{cases}
$$

Note that the operator mixing does not change the value of $n_{Z}=l_{1}+l_{2}$. It turns out that our solution (B.29) can solve these constraints only in limited cases. 

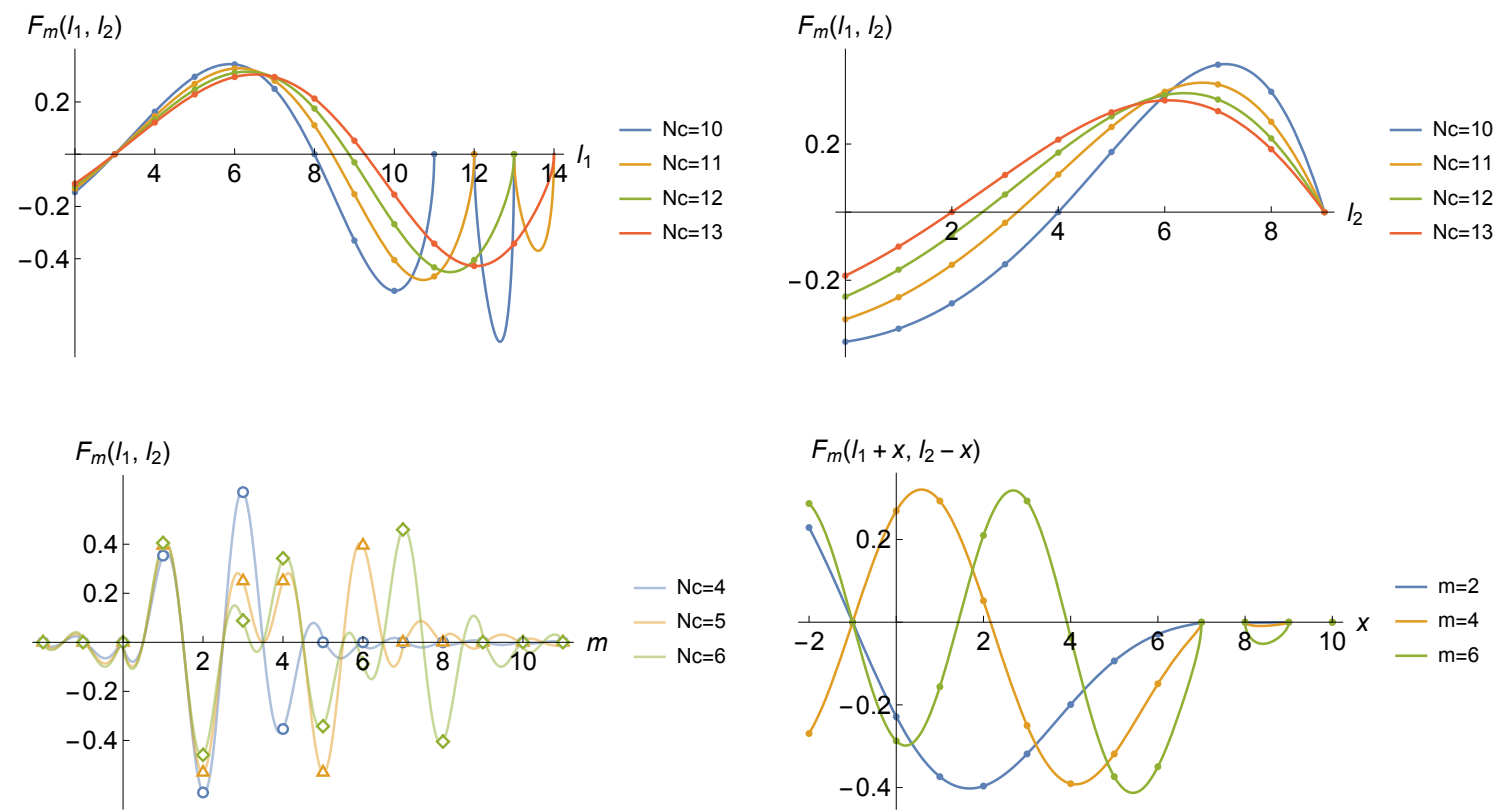

Figure 4. Plotting the function $F_{m}\left(l_{1}, l_{2}\right)$ against $l_{1}, l_{2}$ (above) and $m, x$ (below).

Generally, the function $F_{m}\left(l_{1}, l_{2}\right)$ does not vanish even if $l_{2}<0$, and it slowly decreases to zero when $l_{2}$ is large and negative. This function has a special zero at ${ }^{22}$

$$
0=F_{2 m^{\prime}}(l, l+1) \quad\left(l, m^{\prime} \in \mathbb{Z}_{\geq 0}\right)
$$

meaning that we can solve the Young diagram constraints if all of the following three conditions are satisfied,

- The mode number $m$ is even,

- $n_{Z}=l_{1}+l_{2}$ is odd,

- $n_{Z} \geq N_{c}$.

The last condition may circumvented by using the translation symmetry of the recursion relation $^{23}$

$$
\left(l_{1}, l_{2}, N_{c}\right) \rightarrow\left(l_{1}+x, l_{2}+x, N_{c}+x\right)
$$

although this operation changes the value of $N_{c}$. See figure 4 for the behavior of $F_{m}\left(l_{1}, l_{2}\right)$.

One finds that $F_{m}\left(l_{1}, l_{2}\right)$ for $l_{1}, l_{2}, m \in \mathbb{Z}_{\geq 0}$ satisfies

$$
\begin{array}{ll}
0=F_{m}\left(l_{1}, l_{2}\right) & \left(l_{1}>N_{c}\right) \\
0=F_{m}\left(l_{1}, l_{2}\right) & \left(m<0 \text { or } m>2 N_{c}-n_{Z}+2\right)
\end{array}
$$

\footnotetext{
${ }^{22}$ There are other loci of zeroes, such as $F_{2 m}\left(l_{1}, 1-N_{c}\right)=0$ if $m \leq 2 N_{c}$ and $l_{2} \geq N_{c}-m+2$, which is not meaningful. Practically there is no lower bound for $l_{2}$.

${ }^{23}$ This is different from the symmetry $\left[\mathcal{H}_{1}, \sum_{i=1}^{p} d_{i}^{\dagger}\right]=0$.
} 
as well as (B.32). This suggests that the mode number $m$ in the eigenvalue equation (B.30) should be chosen from

$$
m=2 m^{\prime}, \quad m^{\prime}=1,2, \ldots,\left\lceil N_{c}-\frac{n_{Z}}{2}+1\right\rceil .
$$

We can derive the identities (B.32), (B.34) and (B.35) from the hypergeometric identities in appendix $\mathrm{C}$.

In summary, at $p=2$ we find

$$
D_{1}^{G} O_{F_{2 m^{\prime}}}=8 m^{\prime} n_{12} O_{F_{2 m^{\prime}}}, \quad O_{F_{2 m^{\prime}}}=\sum_{x=-l_{2}}^{\left\lceil\left(l_{1}-l_{2}\right) / 2\right\rceil} F_{2 m^{\prime}}\left(l_{1}-x, l_{2}+x\right) O\left(l_{1}, l_{2}\right)
$$

where $m^{\prime}$ runs over the range (B.36). The result (B.37) reproduces [19], and is valid for any scaling of $\left(l_{1}, l_{2}\right)$ with respect to $N_{c}$. In particular, the eigenvalues are $O(1) \sim O\left(\lambda / N_{c}\right)$ as long as $m \sim O(1)$.

\section{B.3 Examples of the eigenvalues}

If we introduce a reference point 0 so that all edges pass through that point, we can write

$$
n_{i j}=n_{i 0}+n_{j 0} \equiv \tilde{n}_{i}+\tilde{n}_{j}, \quad \tilde{n}_{E} \equiv \sum_{k=1}^{p} \tilde{n}_{k}
$$

where $n_{E}$ is the total number of edges. Then $\mathscr{N}_{i j}$ simplifies a bit,

$$
\mathscr{N}_{i j}=\tilde{n}_{E} \delta_{i j}+\tilde{\mathscr{N}}_{i j}, \quad \tilde{\mathscr{N}}_{i j} \equiv \begin{cases}(p-2) \tilde{n}_{i} & (i=j) \\ -\left(\tilde{n}_{i}+\tilde{n}_{j}\right) & (i \neq j) .\end{cases}
$$

Consider some special cases. The first case is

$$
n_{i}=m,
$$

then

$$
\lambda_{a}= \begin{cases}2 p m & (a=1,2, \ldots p-1) \\ 0 & (a=p)\end{cases}
$$

The residual symmetry is $\mathrm{SO}(p-1)$. The second case is

$$
\tilde{n}_{1} \gg 1, \quad \tilde{n}_{i} \sim O(1) \text { for }(i \neq 1) .
$$

Then

$$
\mathscr{N}_{i j} \sim \begin{cases}(p-1) \tilde{n}_{1} & (i=j=1) \\ \tilde{n}_{1} & (j=j \geq 2) \\ -\tilde{n}_{1} & (i=1, j \neq 1 \text { or } i \neq 1, j=1)\end{cases}
$$

whose eigenvalues are

$$
p \tilde{n}_{1}, \underbrace{\tilde{n}_{1}, \tilde{n}_{1}, \ldots, \tilde{n}_{1}}_{p-2}, 0
$$

neglecting $O(1)$ corrections. The residual symmetry is $\mathrm{SO}(p-2)$. 


\section{Identities of hypergeometric functions}

A special case of Gauss hypergeometric function ${ }_{2} F_{1}$ is called the Kravchuk (or Krawtchouk) polynomial used in [62],

$$
K_{n}(x ; p, q) \equiv{ }_{2} F_{1}(-n,-x ;-q ; 1 / p)=\frac{1}{\left(\begin{array}{c}
n-q-1 \\
n
\end{array}\right)} \sum_{j=0}^{n} p^{-j}\left(\begin{array}{c}
x \\
j
\end{array}\right)\left(\begin{array}{c}
n-q-1 \\
n-j
\end{array}\right)
$$

which satisfies the recursion relation

$$
0=n(1-p) K_{n-1}(x ; p, q)+\{x-n(1-p)-p(q-n)\} K_{n}(x ; p, q)+p(q-n) K_{n+1}(x ; p, q) .
$$

The function ${ }_{2} F_{1}(a, b ; c ; 2)$ is related to ${ }_{2} F_{1}(a, b ; c ;-1)$ by

$$
\begin{aligned}
{ }_{2} F_{1}(a, b ; c ; z)= & \frac{\Gamma(c) \Gamma(c-a-b)}{\Gamma(c-a) \Gamma(c-b)}{ }_{2} F_{1}(a, b ; a+b+1-c ; 1-z) \\
& +\frac{\Gamma(c) \Gamma(a+b-c)}{\Gamma(a) \Gamma(b)}(1-z)^{c-a-b}{ }_{2} F_{1}(c-a, c-b ; 1+c-a-b ; 1-z) .
\end{aligned}
$$

As a corollary,

$$
{ }_{2} F_{1}(-a, b ; c ; z)=\frac{(c-b) a}{(c)_{a}}{ }_{2} F_{1}(-a, b ; b-c-a+1 ; 1-z), \quad(a=0,1,2, \ldots)
$$

where $(x)_{a}=\Gamma(x+a) / \Gamma(x)$. We also have

$$
{ }_{2} F_{1}(a, b ; 1+a-b ;-1)=\frac{\Gamma(1+a-b) \Gamma\left(1+\frac{a}{2}\right)}{\Gamma(1+a) \Gamma\left(1+\frac{a}{2}-b\right)}
$$

Now we can rewrite the rotation matrix (B.22) as

$$
\begin{aligned}
{ }_{1}\left\langle j, j_{1} \mid j, j_{3}\right\rangle= & \frac{(-1)^{j+j_{3}}}{2^{j}} \sqrt{\left(\begin{array}{c}
2 j \\
j+j_{3}
\end{array}\right)\left(\begin{array}{c}
2 j \\
j+j_{1}
\end{array}\right)} \times \\
& \frac{\left(j_{1}-j\right)_{j+j_{3}}}{(-2 j)_{j+j_{3}}}{ }_{2} F_{1}\left(-j-j_{3},-j-j_{1} ;-j_{3}-j_{1}+1 ;-1\right) .
\end{aligned}
$$

and (B.29) as

$$
\begin{aligned}
F_{m}\left(l_{1}, l_{2}\right)= & (-1)^{m-1} 2^{-N_{c}+\frac{1}{2}\left(n_{Z}-1\right)} \sqrt{\left(\begin{array}{c}
2 N_{c}-n_{Z}+1 \\
m-1
\end{array}\right)\left(\begin{array}{c}
2 N_{c}-n_{Z}+1 \\
N_{c}-l_{1}
\end{array}\right)} \times \\
& \frac{\left(l_{2}-N_{c}-1\right)_{m-1}}{\left(n_{Z}-2 N_{c}-1\right)_{m-1}}{ }_{2} F_{1}\left(-m+1,-N_{c}+l_{1} ; N_{c}-l_{2}-m+3 ;-1\right) .
\end{aligned}
$$

Note that the original expression in terms of ${ }_{2} F_{1}(a, b ; c ; 2)$ is more suitable for numerical evaluation. By combining the above identities, we find

$$
\begin{aligned}
F_{2 m^{\prime}}(s, s+1)= & 2^{-3 N_{c}-2+2 m^{\prime}+3 s} \sqrt{\left(\begin{array}{c}
2 N_{c}-2 s \\
N_{c}-s
\end{array}\right)\left(\begin{array}{c}
2 N_{c}-2 s \\
2 m^{\prime}-1
\end{array}\right)} \times \\
& \frac{\Gamma\left(s-N_{c}+\frac{1}{2}\right) \Gamma\left(-N_{c}-1+2 m^{\prime}+s\right) \Gamma\left(N_{c}+2-2 m^{\prime}-s\right)}{\Gamma\left(1-m^{\prime}\right) \Gamma\left(-2 N_{c}-1+2 m^{\prime}+2 s\right) \Gamma\left(N_{c}+\frac{3}{2}-m^{\prime}-s\right)}
\end{aligned}
$$

which vanishes at $m^{\prime}=0,1,2, \ldots$ due to $\Gamma\left(1-m^{\prime}\right)=\infty$. 


\section{Details of strong coupling analysis}

\section{D.1 Geometry}

We parametrize $S^{5}$ by using the embedding coordinates as

$$
\begin{aligned}
& X_{1}=R / \sqrt{\rho} \cos \eta \cos \theta_{1} \\
& X_{2}=R / \sqrt{\rho} \cos \eta \sin \theta_{1} \\
& X_{3}=R / \sqrt{\rho} \sin \eta \cos \theta_{2} \\
& X_{4}=R / \sqrt{\rho} \sin \eta \sin \theta_{2} \\
& X_{5}=R \sqrt{1-1 / \rho} \cos \phi \\
& X_{6}=R \sqrt{1-1 / \rho} \sin \phi
\end{aligned}
$$

where $\rho \geq 1, \eta \in[0, \pi / 2]$ and $\phi, \theta_{1}, \theta_{2} \in[0,2 \pi)$. The polar coordinates on $\mathrm{S}^{3}$ spanned by $\left(X^{1}, X^{2}, X^{3}, X^{4}\right)$ make the symmetry of $\mathrm{SO}(4)=\mathrm{SU}(2) \times \mathrm{SU}(2)$ manifest. The metric on $\mathbb{R}_{t} \times S^{5}$ is

$$
d s^{2}=R^{2}\left\{-d t^{2}+\frac{d \rho^{2}}{4(\rho-1) \rho^{2}}+\frac{(\rho-1) d \phi^{2}}{\rho}+\frac{d \eta^{2}+\cos ^{2} \eta d \theta_{1}^{2}+\sin ^{2} \eta d \theta_{2}^{2}}{\rho}\right\}
$$

and the Laplacian is

$$
\begin{aligned}
\Delta & =\frac{1}{R^{2}}\left\{-\frac{\partial^{2}}{\partial t^{2}}+4(\rho-1) \rho^{2} \frac{\partial^{2}}{\partial \rho^{2}}+4 \rho \frac{\partial}{\partial \rho}+\frac{\rho}{\rho-1} \frac{\partial^{2}}{\partial \phi^{2}}+\frac{\rho}{R^{2}} \Delta_{\mathrm{S}^{3}}\right\} \\
\Delta_{\mathrm{S}^{3}} & =\frac{\partial^{2}}{\partial \eta^{2}}+2 \cot (2 \eta) \frac{\partial}{\partial \eta}+\frac{1}{\cos ^{2} \eta} \frac{\partial^{2}}{\partial \theta_{1}^{2}}+\frac{1}{\sin ^{2} \eta} \frac{\partial^{2}}{\partial \theta_{2}^{2}} .
\end{aligned}
$$

The Jacobian is

$$
d \Omega_{5}=\frac{R^{5} \sin (2 \eta)}{4 \rho^{3}} d \rho d \phi d \eta d \theta_{1} d \theta_{2}
$$

and thus

$$
T_{3} \int_{\Sigma_{4}} \frac{R^{5} \sin (2 \eta)}{2 \rho^{2}} d \phi d \eta d \theta_{1} d \theta_{2}=N_{c} \int_{\mathbb{R}} d t \frac{\partial_{t} \phi}{\rho^{2}}
$$

where (4.3) is used. The RR four-form satisfies $d C_{4} \propto\left(d \Omega_{5}+* d \Omega_{5}\right)$, and the normalization is chosen so that the BPS configuration satisfies $E=J$.

\section{D.2 Spherical harmonics}

The scalar spherical harmonics on $\mathrm{S}^{3}$ is $[64,65]$

$$
\Delta_{\mathrm{S}^{3}} \Phi_{k, m_{1}, m_{2}}=-k(k+2) \Phi_{k, m_{1}, m_{2}}
$$

where $\Delta_{\mathrm{S}^{3}}$ is given in (D.3) and

$$
\begin{aligned}
\Phi_{k, m_{1}, m_{2}}\left(\eta, \theta_{1}, \theta_{2}\right) & =C_{k, m_{1}, m_{2}}\left(e^{i \theta_{1}} \cos \eta\right)^{m_{1}-m_{2}}\left(e^{i \theta_{2}} \sin \eta\right)^{m_{1}+m_{2}} P_{k / 2-m_{1}}^{m_{1}+m_{2}, m_{1}-m_{2}}(\cos 2 \eta) \\
C_{k, m_{1}, m_{2}} & =\sqrt{\frac{k+1}{2 \pi^{2}}} \sqrt{\frac{\left(k / 2+m_{1}\right) !\left(k / 2-m_{1}\right) !}{\left(k / 2+m_{2}\right) !\left(k / 2-m_{2}\right) !}}, \quad \frac{k}{2}-m_{i}=0,1, \ldots, k, \quad k \in \mathbb{Z}_{\geq 0}
\end{aligned}
$$


and $P_{n}^{a, b}(x)$ is the Jacobi polynomial. ${ }^{24}$ Note that $\left\{m_{i}\right\}$ are half-integers when $k$ is odd, and $\Phi_{k, m_{1}, m_{2}}$ is complex-valued,

$$
\left(\Phi_{k, m_{1}, m_{2}}\right)^{*}=(-1)^{m_{1}+m_{2}} \Phi_{k,-m_{1},-m_{2}} .
$$

The spherical harmonics satisfy the orthogonality

$$
\int_{0}^{2 \pi} d \theta_{1} \int_{0}^{2 \pi} d \theta_{2} \int_{0}^{\pi / 2} d \eta \frac{\sin 2 \eta}{2}(-1)^{m_{1}+m_{2}} \Phi_{k, m_{1}, m_{2}} \Phi_{l,-n_{1},-n_{2}}=\delta_{l, k} \delta_{m_{1}, n_{1}} \delta_{m_{2}, n_{2}}
$$

and the integrated spherical harmonics satisfy

$$
\begin{aligned}
& \int_{0}^{2 \pi} d \theta_{1} \int_{0}^{2 \pi} d \theta_{2} \int_{0}^{\pi / 2} d \eta \frac{\sin 2 \eta}{2}\left\{\left(\partial_{\eta} \Phi_{k, m_{1}, m_{2}}\right)\left(\partial_{\eta} \Phi_{l,-n_{1},-n_{2}}\right)+\frac{\left(\partial_{\theta_{1}} \Phi_{k, m_{1}, m_{2}}\right)\left(\partial_{\theta_{1}} \Phi_{l,-n_{1},-n_{2}}\right)}{\cos ^{2} \eta}\right. \\
& \left.+\frac{\left(\partial_{\theta_{2}} \Phi_{k, m_{1}, m_{2}}\right)\left(\partial_{\theta_{2}} \Phi_{l,-n_{1},-n_{2}}\right)}{\sin ^{2} \eta}\right\}=k(k+2) \delta_{l, k} \delta_{m_{1}, n_{1}} \delta_{m_{2}, n_{2}} .
\end{aligned}
$$

The first few eigenfunctions are

$$
\begin{aligned}
& \Phi_{0,0,0}=\frac{1}{\sqrt{2 \pi^{2}}}, \quad \Phi_{1, \frac{1}{2}, \frac{1}{2}}=\frac{e^{i \theta_{2}} \sin \eta}{\pi}, \quad \Phi_{1, \frac{1}{2},-\frac{1}{2}}=\frac{e^{i \theta_{1}} \cos \eta}{\pi}, \\
& \Phi_{2,0,0}=\sqrt{\frac{3}{2 \pi^{2}}} \cos (2 \eta), \quad \Phi_{2,1,0}=\sqrt{\frac{3}{4 \pi^{2}}} e^{i\left(\theta_{1}+\theta_{2}\right)} \sin (2 \eta), \quad \Phi_{2,0,1}=\sqrt{\frac{3}{4 \pi^{2}}} e^{i\left(\theta_{1}-\theta_{2}\right)} \sin (2 \eta) .
\end{aligned}
$$

The functions $\Phi_{2 \ell, 0,0}$ are equal to the Legendre polynomial $P_{\ell}(\cos 2 \eta)$.

\section{D.3 Classical solutions at $j=0,1$}

Consider the classical D3-brane solutions around $j=0,1$, or equivalently $J=0, N_{c} / g_{s}$. For this purpose, we use the coordinate $r=1 / \sqrt{\rho}$ with $0 \leq r \leq 1$. The metric (D.2) becomes

$$
d s^{2}=R^{2}\left\{-d t^{2}+\frac{d r^{2}}{1-r^{2}}+\left(1-r^{2}\right) d \phi^{2}+r^{2}\left(d \eta^{2}+\cos ^{2} \eta d \theta_{1}^{2}+\sin ^{2} \eta d \theta_{2}^{2}\right)\right\} .
$$

We expand the equations of motion around

$$
r=\sqrt{j}+\epsilon r_{1}(t, \eta), \quad \phi=t+\epsilon \phi_{1}(t, \eta), \quad(j=0,1) .
$$

First, consider the case $j=1$. The EoM for $\phi$ becomes $\partial_{t} r_{1}=0$. If we write $r_{1} \equiv$ $-e^{-s(\eta)}$, the EoM for $r$ becomes

$$
\begin{aligned}
0= & \frac{1}{4}\left(s^{\prime 2}-4 \cot (2 \eta) s^{\prime}-2 s^{\prime \prime}\right) \\
& -\epsilon\left\{2 \partial_{t} \phi_{1}-\frac{e^{-s(\eta)}}{16}\left(6 s^{\prime \prime}\left(s^{\prime 2}+2\right)-3 s^{\prime 2}\left(s^{\prime 2}+4\right)+4 \cot (2 \eta) s^{\prime}\left(s^{\prime 2}+6\right)\right)\right\}+O\left(\epsilon^{2}\right) .
\end{aligned}
$$

\footnotetext{
${ }^{24}$ Our convention is same as JacobiP $[\mathrm{n}, \mathrm{a}, \mathrm{b}, \mathrm{x}]$ in Mathematica.
} 
The general solution at $O\left(\epsilon^{0}\right)$ is

$$
e^{-s(\eta)}=\left(\frac{c_{(2)}+c_{(1)} \log \cot \eta}{4}\right)^{2} .
$$

To maintain $r \in[0,1]$ we need $c_{(1)}=0$. Then, the equation (D.14) produces $\partial_{t} \phi_{1}=0$. Thus there is no non-trivial solution.

Next, consider the case $j=0$. The EoM's give us

$$
0=r_{1}\left(\cot (2 \eta) r_{1}+\partial_{\eta} r_{1}\right)=r_{1} \partial_{\eta} \phi_{1}
$$

The non-trivial solution of the first equation is

$$
r_{1}=\frac{c}{\sqrt{\sin 2 \eta}}
$$

for a constant $c$. This function diverges around $\eta=0, \pi / 2$, and is inconsistent with $r \in[0,1]$. Again, we find no non-trivial solution.

\section{E On non-abelian DBI}

The symmetry of the effective $\mathrm{U}(p)$ theory becomes non-abelian if some of the column lengths become equal, $l_{i}=l_{i+1}$ for some $i$ in (A.9). Correspondingly, some of the $p$ giant graviton branes coincide at strong coupling. Let us make a short digression about nonabelian DBI action to examine this situation. ${ }^{25}$

The DBI action is a low energy effective action of closed and open string massless modes on the brane. As a worldvolume theory, the DBI action without $\mathrm{U}(1)$ flux is also an example of $4 \mathrm{~d}$ conformal theory. In $\mathrm{AdS}_{5} \times \mathrm{S}^{5}$, the conformal symmetry of the target spacetime is nonlinearly realized [66, 67]. The DBI action can be made supersymmetric in the sense of $\kappa$ symmetry [44, 68, 69] and of the worldvolume symmetry [70, 71]. The fundamental strings can be coupled to multiple coincident D-branes by introducing nonabelian flux $F_{\mu \nu}$ [72]. The addition of the CS term to the non-abelian DBI induces dielectric effects [49].

One way to define non-abelian DBI is to expand the DBI action in a formal series of $F$,

$$
\operatorname{det}\left(G+2 \pi \alpha^{\prime} F\right)=\operatorname{det} G\left(1-\frac{\left(2 \pi \alpha^{\prime}\right)^{2}}{2} G^{a b} F_{b c} G^{c d} F_{d a}+\ldots\right) .
$$

Then we promote $F$ to a non-abelian field, and take the trace. This procedure suffers from the ordering ambiguity, which should be fixed by the consistency with the open string amplitude [73-76].

In section 4, we want to find classical solutions continuously connected to the spherical giant graviton. From the above prescription for the non-abelian DBI in (E.1), we do not see any significant difference between $\mathrm{U}(1)^{p}$ and $\mathrm{U}(p)$ theories at the order of $\alpha^{\prime 2}$.

\footnotetext{
${ }^{25}$ The author thanks A. Tseytlin for his comments on abelian and non-abelian DBI.
} 
The induced metric $G_{a b}$ should not have the $\mathrm{U}(p)$ structure for the following reasons. First, the induced metric comes from closed string massless modes, which do not see nonabelian symmetry. Second, the attempts to make $G_{a b}$ a matrix-valued field suffer from various difficulties $[77,78]$. Third, in our deformation problem, we are only interested in the corrections of $O\left(\alpha^{\prime 2}\right)$, and the commutator terms do not show up at this order. Then, all matrices are simultaneously diagonalizable, unless they couple to other objects.

Open Access. This article is distributed under the terms of the Creative Commons Attribution License (CC-BY 4.0), which permits any use, distribution and reproduction in any medium, provided the original author(s) and source are credited.

\section{References}

[1] J.M. Maldacena, The Large $N$ limit of superconformal field theories and supergravity, Adv. Theor. Math. Phys. 2 (1998) 231 [hep-th/9711200] [InSPIRE].

[2] D.J. Binder, S.M. Chester, S.S. Pufu and Y. Wang, $\mathcal{N}=4$ Super-Yang-Mills correlators at strong coupling from string theory and localization, JHEP 12 (2019) 119 [arXiv:1902.06263] [INSPIRE].

[3] H. Lin, O. Lunin and J.M. Maldacena, Bubbling AdS space and 1/2 BPS geometries, JHEP 10 (2004) 025 [hep-th/0409174] [INSPIRE].

[4] J. McGreevy, L. Susskind and N. Toumbas, Invasion of the giant gravitons from Anti-de Sitter space, JHEP 06 (2000) 008 [hep-th/0003075] [INSPIRE].

[5] R. de Mello Koch, C. Mathwin and H.J.R. van Zyl, LLM Magnons, JHEP 03 (2016) 110 [arXiv: 1601.06914] [INSPIRE].

[6] R. de Mello Koch, M. Kim and H.J.R. Zyl, Integrable Subsectors from Holography, JHEP 05 (2018) 198 [arXiv: 1802.01367] [INSPIRE].

[7] R. de Mello Koch, J.-H. Huang and L. Tribelhorn, Exciting LLM Geometries, JHEP 07 (2018) 146 [arXiv: 1806. 06586] [inSPIRE].

[8] D. Berenstein and A. Holguin, Open giant magnons on LLM geometries, JHEP 01 (2021) 080 [arXiv: 2010.02236$]$ [INSPIRE].

[9] R. Suzuki, Three-point functions in $\mathcal{N}=4 S Y M$ at finite $N_{c}$ and background independence, JHEP 05 (2020) 118 [arXiv:2002.07216] [INSPIRE].

[10] S. Corley, A. Jevicki and S. Ramgoolam, Exact correlators of giant gravitons from dual $\mathcal{N}=4$ SYM theory, Adv. Theor. Math. Phys. 5 (2002) 809 [hep-th/0111222] [INSPIRE].

[11] V. Balasubramanian, D. Berenstein, B. Feng and M.-x. Huang, D-branes in Yang-Mills theory and emergent gauge symmetry, JHEP 03 (2005) 006 [hep-th/0411205] [INSPIRE].

[12] R. de Mello Koch, J. Smolic and M. Smolic, Giant Gravitons - with Strings Attached (I), JHEP 06 (2007) 074 [hep-th/0701066] [INSPIRE].

[13] V. De Comarmond, R. de Mello Koch and K. Jefferies, Surprisingly Simple Spectra, JHEP 02 (2011) 006 [arXiv: 1012.3884] [INSPIRE].

[14] W. Carlson, R. de Mello Koch and H. Lin, Nonplanar Integrability, JHEP 03 (2011) 105 [arXiv: 1101.5404] [INSPIRE]. 
[15] R. de Mello Koch, M. Dessein, D. Giataganas and C. Mathwin, Giant Graviton Oscillators, JHEP 10 (2011) 009 [arXiv: 1108.2761] [INSPIRE].

[16] R. de Mello Koch and S. Ramgoolam, A double coset ansatz for integrability in AdS/CFT, JHEP 06 (2012) 083 [arXiv: 1204.2153] [INSPIRE].

[17] R. de Mello Koch, B.A.E. Mohammed and S. Smith, Nonplanar Integrability: Beyond the SU(2) Sector, Int. J. Mod. Phys. A 26 (2011) 4553 [arXiv:1106.2483] [inSPIRE].

[18] R. de Mello Koch, P. Diaz and N. Nokwara, Restricted Schur Polynomials for Fermions and integrability in the $\mathrm{SU}(2 \mid 3)$ sector, JHEP 03 (2013) 173 [arXiv:1212.5935] [INSPIRE].

[19] R. de Mello Koch, G. Kemp, B.A.E. Mohammed and S. Smith, Nonplanar integrability at two loops, JHEP 10 (2012) 144 [arXiv:1206.0813] [INSPIRE].

[20] R. de Mello Koch, S. Graham and I. Messamah, Higher Loop Nonplanar Anomalous Dimensions from Symmetry, JHEP 02 (2014) 125 [arXiv: 1312.6227] [INSPIRE].

[21] H. Lin, Relation between large dimension operators and oscillator algebra of Young diagrams, Int. J. Geom. Meth. Mod. Phys. 12 (2015) 1550047 [arXiv: 1407.7815] [InSPIRE].

[22] S. de Carvalho, R. de Mello Koch and A. Larweh Mahu, Anomalous dimensions from boson lattice models, Phys. Rev. D 97 (2018) 126004 [arXiv:1801.02822] [inSPIRE].

[23] R. de Mello Koch, J.-H. Huang, M. Kim and H.J.R. Van Zyl, Emergent Yang-Mills theory, JHEP 10 (2020) 100 [arXiv:2005.02731] [INSPIRE].

[24] J. Polchinski, TASI lectures on D-branes, in Theoretical Advanced Study Institute in Elementary Particle Physics (TASI 96): Fields, Strings, and Duality, 11, 1996 [hep-th/9611050] [INSPIRE].

[25] M.T. Grisaru, R.C. Myers and O. Tafjord, SUSY and goliath, JHEP 08 (2000) 040 [hep-th/0008015] [INSPIRE].

[26] A. Hashimoto, S. Hirano and N. Itzhaki, Large branes in AdS and their field theory dual, JHEP 08 (2000) 051 [hep-th/0008016] [INSPIRE].

[27] S.R. Das, A. Jevicki and S.D. Mathur, Vibration modes of giant gravitons, Phys. Rev. D 63 (2001) 024013 [hep-th/0009019] [INSPIRE].

[28] C.G. Callan and J.M. Maldacena, Brane death and dynamics from the Born-Infeld action, Nucl. Phys. B 513 (1998) 198 [hep-th/9708147] [INSPIRE].

[29] D. Sadri and M.M. Sheikh-Jabbari, Giant hedgehogs: Spikes on giant gravitons, Nucl. Phys. B 687 (2004) 161 [hep-th/0312155] [INSPIRE].

[30] S. Hirano, Fat magnon, JHEP 04 (2007) 010 [hep-th/0610027] [INSPIRE].

[31] A. Dekel and Y. Oz, Integrability of Green-Schwarz Sigma Models with Boundaries, JHEP 08 (2011) 004 [arXiv: 1106.3446] [INSPIRE].

[32] D.M. Hofman and J.M. Maldacena, Reflecting magnons, JHEP 11 (2007) 063 [arXiv:0708.2272] [INSPIRE].

[33] D. Berenstein, Giant gravitons: a collective coordinate approach, Phys. Rev. D 87 (2013) 126009 [arXiv: 1301.3519] [INSPIRE].

[34] D. Berenstein and E. Dzienkowski, Open spin chains for giant gravitons and relativity, JHEP 08 (2013) 047 [arXiv: 1305.2394] [INSPIRE]. 
[35] D. Berenstein, On the central charge extension of the $\mathcal{N}=4$ SYM spin chain, JHEP 05 (2015) 129 [arXiv: 1411.5921] [INSPIRE].

[36] D.M. Hofman and J.M. Maldacena, Giant Magnons, J. Phys. A 39 (2006) 13095 [hep-th/0604135] [INSPIRE].

[37] N. Beisert, C. Kristjansen and M. Staudacher, The Dilatation operator of conformal $\mathcal{N}=4$ superYang-Mills theory, Nucl. Phys. B 664 (2003) 131 [hep-th/0303060] [INSPIRE].

[38] N. Beisert, T. McLoughlin and R. Roiban, The Four-loop dressing phase of $\mathcal{N}=4 S Y M$, Phys. Rev. D 76 (2007) 046002 [arXiv:0705.0321] [InSPIRE].

[39] F. Fiamberti, A. Santambrogio and C. Sieg, Five-loop anomalous dimension at critical wrapping order in $\mathcal{N}=4$ SYM, JHEP 03 (2010) 103 [arXiv:0908.0234] [INSPIRE].

[40] R. de Mello Koch, G. Kemp and S. Smith, From Large $N$ Nonplanar Anomalous Dimensions to Open Spring Theory, Phys. Lett. B 711 (2012) 398 [arXiv:1111.1058] [InSPIRE].

[41] R. de Mello Koch, S. Graham and W. Mabanga, Subleading corrections to the Double Coset Ansatz preserve integrability, JHEP 02 (2014) 079 [arXiv:1312.6230] [INSPIRE].

[42] N. Beisert, The SU(2|2) dynamic S-matrix, Adv. Theor. Math. Phys. 12 (2008) 945 [hep-th/0511082] [INSPIRE].

[43] C. Kristjansen, J. Plefka, G.W. Semenoff and M. Staudacher, A New double scaling limit of $\mathcal{N}=4$ superYang-Mills theory and PP wave strings, Nucl. Phys. B 643 (2002) 3 [hep-th/0205033] [INSPIRE].

[44] R.R. Metsaev and A.A. Tseytlin, Supersymmetric D3-brane action in $A d S_{5} \times S^{5}$, Phys. Lett. B 436 (1998) 281 [hep-th/9806095] [INSPIRE].

[45] S. de Carvalho, R. de Mello Koch and M. Kim, Central Charges for the Double Coset, JHEP 05 (2020) 007 [arXiv: 2001.10181] [INSPIRE].

[46] D.H. Correa and C.A.S. Young, Finite size corrections for open strings/open chains in planar AdS/CFT, JHEP 08 (2009) 097 [arXiv: 0905.1700] [INSPIRE].

[47] R. de Mello Koch, P. Diaz and H. Soltanpanahi, Non-planar Anomalous Dimensions in the SL(2) Sector, Phys. Lett. B 713 (2012) 509 [arXiv:1111.6385] [InSPIRE].

[48] A. Mohamed Adam Ali, R. de Mello Koch, N.H. Tahiridimbisoa and A. Larweh Mahu, Interacting Double Coset Magnons, Phys. Rev. D 93 (2016) 065057 [arXiv:1512.05019] [INSPIRE].

[49] R.C. Myers, Dielectric branes, JHEP 12 (1999) 022 [hep-th/9910053] [INSPIRE].

[50] A. Hashimoto, The Shape of branes pulled by strings, Phys. Rev. D 57 (1998) 6441 [hep-th/9711097] [INSPIRE].

[51] N.R. Constable, R.C. Myers and O. Tafjord, The Noncommutative bion core, Phys. Rev. D 61 (2000) 106009 [hep-th/9911136] [INSPIRE].

[52] M.M. Sheikh-Jabbari, Tiny graviton matrix theory: DLCQ of IIB plane-wave string theory, a conjecture, JHEP 09 (2004) 017 [hep-th/0406214] [INSPIRE].

[53] M.M. Sheikh-Jabbari and M. Torabian, Classification of all 1/2 BPS solutions of the tiny graviton matrix theory, JHEP 04 (2005) 001 [hep-th/0501001] [INSPIRE]. 
[54] M. Ali-Akbari, M.M. Sheikh-Jabbari and M. Torabian, Tiny Graviton Matrix Theory/SYM Correspondence: Analysis of BPS States, Phys. Rev. D 74 (2006) 066005 [hep-th/0606117] [INSPIRE].

[55] A. Bissi, C. Kristjansen, D. Young and K. Zoubos, Holographic three-point functions of giant gravitons, JHEP 06 (2011) 085 [arXiv:1103.4079] [INSPIRE].

[56] H. Lin, Giant gravitons and correlators, JHEP 12 (2012) 011 [arXiv: 1209.6624] [INSPIRE].

[57] P. Caputa, R. de Mello Koch and K. Zoubos, Extremal versus Non-Extremal Correlators with Giant Gravitons, JHEP 08 (2012) 143 [arXiv: 1204.4172] [INSPIRE].

[58] J. Caetano, W. Peelaers and L. Rastelli, Maximally Supersymmetric RG Flows in $4 D$ and Integrability, arXiv:2006.04792 [INSPIRE].

[59] K. Costello, E. Witten and M. Yamazaki, Gauge Theory and Integrability, I, ICCM Not. 06 (2018) 46 [arXiv: 1709.09993] [INSPIRE].

[60] K. Costello, E. Witten and M. Yamazaki, Gauge Theory and Integrability, II, ICCM Not. 06 (2018) 120 [arXiv : 1802.01579] [INSPIRE].

[61] K. Costello and M. Yamazaki, Gauge Theory And Integrability, III, arXiv:1908.02289 [INSPIRE].

[62] N. Atakishiyev, G. Pogosyan and K. Wolf, Finite models of the oscillator, Fiz. Elem. Chastits At. Yadra 36 (2005) 473.

[63] E.I. Jafarov, N.I. Stoilova and J. Van der Jeugt, Finite oscillator models: The Hahn oscillator, J. Phys. A 44 (2011) 265203 [arXiv:1101.5310] [INSPIRE].

[64] M. Bander and C. Itzykson, Group Theory and the Hydrogen Atom (I), Rev. Mod. Phys. 38 (1966) 330.

[65] M. Lachieze-Rey and S. Caillerie, Laplacian eigenmodes for spherical spaces, Class. Quant. Grav. 22 (2005) 695 [astro-ph/0501419] [INSPIRE].

[66] A. Jevicki, Y. Kazama and T. Yoneya, Quantum metamorphosis of conformal transformation in D3-brane Yang-Mills theory, Phys. Rev. Lett. 81 (1998) 5072 [hep-th/9808039] [InSPIRE].

[67] S.M. Kuzenko and I.N. McArthur, Quantum metamorphosis of conformal symmetry in $\mathcal{N}=4$ super Yang-Mills theory, Nucl. Phys. B 640 (2002) 78 [hep-th/0203236] [INSPIRE].

[68] M. Aganagic, C. Popescu and J.H. Schwarz, D-brane actions with local kappa symmetry, Phys. Lett. B 393 (1997) 311 [hep-th/9610249] [INSPIRE].

[69] M. Aganagic, C. Popescu and J.H. Schwarz, Gauge invariant and gauge fixed D-brane actions, Nucl. Phys. B 495 (1997) 99 [hep-th/9612080] [INSPIRE].

[70] M. Roček and A.A. Tseytlin, Partial breaking of global D $=4$ supersymmetry, constrained superfields, and three-brane actions, Phys. Rev. D 59 (1999) 106001 [hep-th/9811232] [INSPIRE].

[71] A.A. Tseytlin, Born-Infeld action, supersymmetry and string theory, hep-th/9908105 [INSPIRE].

[72] A.A. Tseytlin, On nonAbelian generalization of Born-Infeld action in string theory, Nucl. Phys. B 501 (1997) 41 [hep-th/9701125] [INSPIRE].

[73] R. Medina, F.T. Brandt and F.R. Machado, The Open superstring five point amplitude revisited, JHEP 07 (2002) 071 [hep-th/0208121] [INSPIRE]. 
[74] L.A. Barreiro and R. Medina, 5-field terms in the open superstring effective action, JHEP 03 (2005) 055 [hep-th/0503182] [INSPIRE].

[75] D. Oprisa and S. Stieberger, Six gluon open superstring disk amplitude, multiple hypergeometric series and Euler-Zagier sums, hep-th/0509042 [INSPIRE].

[76] J. Broedel, O. Schlotterer and S. Stieberger, Polylogarithms, Multiple Zeta Values and Superstring Amplitudes, Fortsch. Phys. 61 (2013) 812 [arXiv:1304.7267] [InSPIRE].

[77] M.R. Douglas, D-branes and matrix theory in curved space, Nucl. Phys. B Proc. Suppl. 68 (1998) 381 [hep-th/9707228] [INSPIRE].

[78] J. De Boer and K. Schalm, General covariance of the nonAbelian DBI action, JHEP 02 (2003) 041 [hep-th/0108161] [INSPIRE]. 